Document downloaded from:

http://hdl.handle.net/10251/166346

This paper must be cited as:

Mastylo, M.; Sánchez Pérez, EA. (2020). Factorization theorems for some new classes of multilinear operators. Asian Journal of Mathematics. 24(1):1-30.

https://doi.org/10.4310/AJM.2020.v24.n1.a1

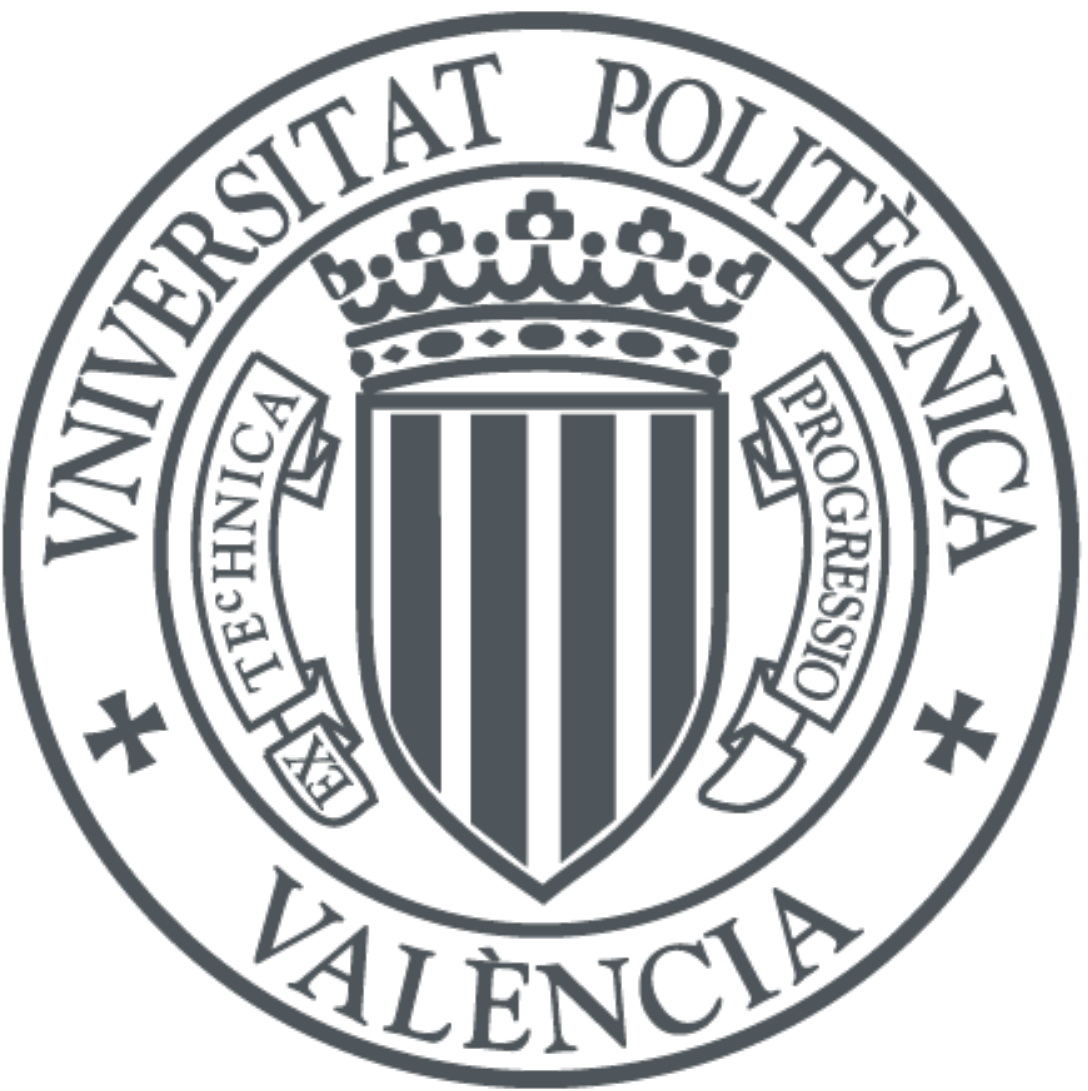

The final publication is available at

https://doi.org/10.4310/AJM.2020.v24.n1.a1

Copyright International Press

Additional Information 


\title{
FACTORIZATION THEOREMS FOR SOME NEW CLASSES OF MULTILINEAR OPERATORS
}

\author{
M. MASTYŁO AND E. A. SÁNCHEZ PÉREZ
}

\begin{abstract}
Two new classes of summing multilinear operators, factorable $(q, p)$-summing operators and $(r ; p, q)$-summing operators are studied. These classes are described in terms of factorization. It is shown that operators in the first (resp., the second) class admit the factorization through the injective tensor product of Banach spaces (resp., through some Banach lattices). Applications in different contexts related to Grothendieck Theorem and Fourier integral bilinear operators are shown. Motivated by Pisier's Theorem on factorization of $(q, 1)$-summing operators from $C(K)$-spaces through Lorentz spaces $L_{q, 1}$ on some probability Borel measure spaces, we prove two variants of Pisier's Theorem for bilinear operators on the product of $C(K)$-spaces. We also prove bilinear versions of Mityagin-Pełczyński and Kislyakov Theorems.
\end{abstract}

\section{INTRODUCTION}

The concept of absolutely $p$-summing operators due to Pietsch [28] and the notion of $(q, p)$ summing operator due to Mityagin and Pełczyński [26] play a fundamental role in the theory of Banach operator ideals; $(q, p)$-summing operators found deep applications in many areas of modern functional analysis including for example eigenvalue distribution of Riesz operators (see $[10,15,17])$. A particular role is played by factorization theorems. Besides, there are many different concrete applications of such factorization that arise in different contexts. We mention famous Pietsch's as well as Pisier's Factorization Theorems for linear operators. In recent years several attempts have been made to generalize classical linear theory to various multilinear settings; we refer to the survey paper [25] and references therein.

The purpose of this paper is to analyze classes of multilinear operators with new types of summability and to prove new factorization theorems for them. These spaces of operators are defined to fit with the main known results on summing linear operators on $C(K)$ spaces, and so their properties are closely related to their behavior when acting in products of $C(K)$ spaces. Motivated in part by the importance of Pisier's Theorem on factorization of $(q, 1)$ summing operators on $C(K)$ spaces through Lorentz $L_{q, 1}(\lambda)$ spaces, we will provide two possible extensions of this result in the multilinear setting.

The paper is organized as follows. In Section 2 we investigate the class of multilinear operators that are defined as the composition of pointwise products of functions and a summing linear operator. After giving a general factorization theorem, some applications are given in four subsections. In particular, a bilinear version of Pisier's Theorem is proved, which gives a characterization of bilinear operators on products of $C(K)$-spaces that admit factorization

2010 Mathematics Subject Classification. Primary 46E30, Secondary 47B38, 46B42.

Key words and phrases. Bilinear operator, Fourier integral bilinear operators, Factorization, Pisier's Theorem.

The first named author was supported by National Science Centre, Poland, project, no. 2015/17/B/ST1/00064. The second named author was supported by the Ministerio de Economía y Competitividad (Spain) under project MTM2016-77054-C2-1-P (Spain). 
through a Lorentz space by means of the pointwise product of functions belonging to the $C(K)$-spaces appearing in the domain.

Bilinear operators satisfying Grothendieck's Theorem are analyzed in other part of this section, as well as some applications to Fourier bilinear integrals. Finally bilinear versions of Mityagin-Pełczyński and Kislyakov Theorems for factorable $(q, p)$-summing operators are given.

In Section 3 we provide a characterization in terms of factorization through a special type of tensor product of Banach lattices of a class of $(r ; p, q)$-summing Banach space valued bilinear operators from the product of $C(K)$-spaces under the assumption $1 \leq p<r$ and $1 \leq q<r$. We prove that this class coincides with the class of all bilinear operators that factor through a positive $(r ; p, q)$-summing operator. This gives an adequate extension of Pisier's Theorem for linear operators just taking into account that it can be stated in the equivalent form: an operator $T$ from a $C(K)$-space to a Banach space $Y$ is $(q, p)$-summing if and only if it factors through a positive $(q, p)$-summing operator. The main difference with the version of Pisier's Theorem given in Section 2 is that in this case the factorization is given by the composition of two linear maps (first) and a bilinear operator (second); in the one provided in Section 2 , the factorization is first given by a bilinear operator (first) that is in fact a multiplication operator, and (second) a linear map. Therefore, the result provides a essentially different Pisier-type factorization.

In general, notation will be introduced as and when needed. Nevertheless, we remind the reader some standard notation from the Banach space theory and the theory of multilinear operators. Following the concept introduced by Pietsch in [30] for scalar valued multilinear operators, if $0<q, p_{1}, \ldots, p_{n} \leq \infty$ are such that $1 / q \leq 1 / p_{1}+\cdots+1 / p_{n}$, an $n$-linear operator $T: X_{1} \times \cdots \times X_{n} \rightarrow Y$ is said to be $\left(q ; p_{1}, \ldots, p_{n}\right)$-summing if there exists $C>0$ such that

$$
\left(\sum_{j=1}^{m}\left\|T\left(x_{j}^{(1)}, \ldots, x_{j}^{(n)}\right)\right\|_{Y}^{q}\right)^{1 / q} \leq C \prod_{k=1}^{n}\left\|\left(x_{j}^{(k)}\right)_{j=1}^{m}\right\|_{w, p_{k}},
$$

for every choice of finite sequences $\left(x_{j}^{(k)}\right)_{j=1}^{m}$ in $X_{k}, 1 \leq k \leq n$. Here, for a given $0<p<\infty$ and every finite sequence $\left(x_{j}\right)_{j=1}^{m}$ in a Banach space $E$,

$$
\left\|\left(x_{j}\right)_{j=1}^{m}\right\|_{w, p}:=\sup _{\left\|x^{*}\right\|_{E^{*} \leq 1}}\left(\sum_{j=1}^{m}\left|x^{*}\left(x_{j}\right)\right|^{p}\right)^{1 / p} .
$$

Recall that in the linear case (i.e., $n=1$ ) and for $0<p \leq q<\infty$ such an operator $T: X_{1} \rightarrow Y$ is called $(q, p)$-summing ( $p$-summing for short if $q=p$ ).

Our main reference for tensor products and tensor norms is [7]; some ideas from the presentation given in [9] are also taken into account. For the theory of Banach operator ideals we refer to [29]. As usual, given two Banach spaces $X$ and $Y$, we denote by $\varepsilon$ the injective norm on the tensor product $X \otimes Y$ defined by

$$
\varepsilon(u)=\sup \left\{\left|\sum_{j=1}^{n}\left\langle x_{j}, x^{*}\right\rangle\left\langle y_{j}, y^{*}\right\rangle\right| ; x^{*} \in B_{X^{*}}, y^{*} \in B_{Y^{*}}\right\}, \quad u \in X \otimes Y,
$$

where $\sum_{j=1}^{n} x_{j} \otimes y_{j}$ is any representation of $u$. The completion denoted by $X \widehat{\otimes}_{\varepsilon} Y$ is called the injective tensor product of $X$ and $Y$. We define the injective tensor product $X_{1} \widehat{\otimes}_{\varepsilon} \cdots \widehat{\otimes}_{\varepsilon} X_{n}$ of Banach spaces $X_{1}, \ldots, X_{n}$ in the same way.

We will use the well known fact that if $K_{1}$ and $K_{2}$ are two compact Hausdorff spaces then the injective tensor product $C\left(K_{1}\right) \widehat{\otimes}_{\varepsilon} C\left(K_{2}\right)$ of the corresponding spaces of continuous functions can be identified in a natural way with $C\left(K_{1} \times K_{2}\right)$. 
We recall that a Banach space $X$ has cotype $q(2 \leq q<\infty)$ if there is a constant $C>0$ such that no matter how we select the finite sequence $\left(x_{k}\right)_{k=1}^{n}$ in $X$,

$$
\left(\sum_{k=1}^{n}\left\|x_{k}\right\|_{X}^{q}\right)^{1 / q} \leq C\left(\int_{0}^{1}\left\|\sum_{k=1}^{n} r_{k}(t) x_{k}\right\|_{X}^{2} d t\right)^{1 / 2}
$$

where $\left(r_{k}\right)$ is the sequence of Rademacher functions. The smallest of all these constants is denoted by $C_{q}(X)$.

\section{FaCtorable $(q, p)$-Summing multilinear operators}

In this section we initiate the study of factorization for some new classes of multilinear operators. Our primary motivation for studying these classes stems out from the fact that some known important multilinear operators belong to these classes. We begin with a key definition.

Definition 2.1. Let $X_{1}, \ldots, X_{n}, Y$ be Banach spaces, and let $1 \leq q, p<\infty$. An $n$-linear operator $T: X_{1} \times \cdots \times X_{n} \rightarrow Y$ is said to be factorable $(q, p)$-summing (with constant $C>0$ ) if there exists $C>0$ such that for every positive integers $M, N$ and all $M \times N$ matrices $\left(x_{j k}^{(1)}\right), \ldots,\left(x_{j k}^{(n)}\right)$ in $X_{1}, \ldots, X_{n}$, respectively, we have

$$
\begin{aligned}
& \left(\sum_{j=1}^{M}\left\|\sum_{k=1}^{N} T\left(x_{j k}^{(1)}, \ldots, x_{j k}^{(n)}\right)\right\|_{Y}^{q}\right)^{1 / q} \\
& \leq C \sup _{x_{1}^{*} \in B_{X_{1}^{*}, \ldots, x_{n}^{*} \in B_{X_{n}^{*}}}}\left(\sum_{j=1}^{M}\left|\sum_{k=1}^{N}\left\langle x_{j k}^{(1)}, x_{1}^{*}\right\rangle \cdots\left\langle x_{j k}^{(n)}, x_{n}^{*}\right\rangle\right|^{p}\right)^{1 / p} .
\end{aligned}
$$

A simple observation is that we may replace the balls $B_{X_{k}^{*}}$ in the above formula by norming sets. Recall that if $X$ is a Banach space, then a subset $E \subset B_{X^{*}}$ is said to be norming whenever $\|x\|_{X}=\sup \left\{\left|x^{*}(x)\right| ; x^{*} \in E\right\}$ for every $x \in X$.

Definition 2.1 is motivated in part by the fact that Pietsch-integral bilinear operators are factorable $(q, p)$-summing for any $1 \leq q, p<\infty$. To see this we first recall that a bilinear operator $T: X_{1} \times X_{2} \rightarrow Y$ is called Pietsch-integral (P-integral for short, see [1,35]) if there is a regular countably additive, $Y$-valued Borel measure $\nu$ of bounded variation on $B_{X_{1}^{*}} \times B_{X_{2}^{*}}$ such that

$$
T\left(x^{(1)}, x^{(2)}\right)=\int_{B_{X_{1}^{*} \times B_{X_{2}^{*}}}}\left\langle x^{(1)}, x_{1}^{*}\right\rangle\left\langle x^{(2)}, x_{2}^{*}\right\rangle d \nu\left(x_{1}^{*}, x_{2}^{*}\right), \quad\left(x^{(1)}, x^{(2)}\right) \in X_{1} \times X_{2} .
$$

The following calculations show that such a bilinear operator is factorable $q$-summing for every $1 \leq q<\infty$, and so factorable $(q, p)$-summing for all $1 \leq p \leq q<\infty$. Fix $M \times N$ matrices $\left(x_{j k}^{(1)}\right)$ and $\left(x_{j k}^{(2)}\right)$ in $X_{1}$ and $X_{2}$, respectively and suppose that $T: X_{1} \times X_{2} \rightarrow Y$ is a Pietsch-integral bilinear operator associated to the regular Borel measure $\nu$. Write $|\nu|$ for the variation of the measure. Using the properties of the vector-valued integral, we obtain 
the following estimates with $C=|\nu|\left(B_{X_{1}^{*}} \times B_{X_{2}^{*}}\right)$,

$$
\begin{aligned}
& \left.\left(\sum_{j=1}^{M}\left\|\sum_{k=1}^{N} T\left(x_{j k}^{(1)}, x_{j k}^{(2)}\right)\right\|_{Y}^{q}\right)^{1 / q}=\left(\sum_{j=1}^{M} \| \int_{B_{X_{1}^{*}} \times B_{X_{2}^{*}}}\left(\sum_{k=1}^{N}\left\langle x_{j k}^{(1)}, x_{1}^{*}\right\rangle\left\langle x_{j k}^{(2)}\right), x_{2}^{*}\right\rangle\right) d \nu \|_{Y}^{q}\right)^{1 / q} \\
& \leq\left(\sum_{j=1}^{M}\left(\int_{B_{X_{1}^{*}} \times B_{X_{2}^{*}}}\left|\sum_{k=1}^{N}\left\langle x_{j k}^{(1)}, x_{1}^{*}\right\rangle\left\langle x_{j k}^{(2)}, x_{2}^{*}\right\rangle\right| d|\nu|\right)^{q}\right)^{1 / q} \\
& =\sup _{\left(\lambda_{j}\right) \in B_{\ell^{q^{\prime}}}}\left(\sum_{j=1}^{M} \lambda_{j} \int_{B_{X_{1}^{*}} \times B_{X_{2}^{*}}}\left|\sum_{k=1}^{N}\left\langle x_{j k}^{(1)}, x_{1}^{*}\right\rangle\left\langle x_{j k}^{(2)}, x_{2}^{*}\right\rangle\right| d|\nu|\right) \\
& \leq \int_{B_{X_{1}^{*} \times B_{X_{2}^{*}}}} \sup _{\left(\lambda_{j}\right) \in B_{\ell q^{\prime}}}\left(\sum_{j=1}^{M} \lambda_{j}\left|\sum_{k=1}^{N}\left\langle x_{j k}^{(1)}, x_{1}^{*}\right\rangle\left\langle x_{j k}^{(2)}, x_{2}^{*}\right\rangle\right|\right) d|\nu| \\
& \leq C \sup _{\left(x_{1}^{*}, x_{2}^{*}\right) \in B_{X_{1}^{*}} \times B_{X_{2}^{*}}} \sup _{\left(\lambda_{j}\right) \in B_{\ell^{\prime}}}\left(\sum_{j=1}^{M} \lambda_{j}\left|\sum_{k=1}^{N}\left\langle x_{j k}^{(1)}, x_{1}^{*}\right\rangle\left\langle x_{j k}^{(2)}, x_{2}^{*}\right\rangle\right|\right) \\
& =C \sup _{x_{1}^{*} \in B_{X_{1}^{*}, \ldots, x_{n}^{*} \in B_{X_{n}^{*}}}}\left(\sum_{j=1}^{M}\left|\sum_{k=1}^{N}\left\langle x_{j k}^{(1)}, x_{1}^{*}\right\rangle \cdots\left\langle x_{j k}^{(n)}, x_{n}^{*}\right\rangle\right|^{q}\right)^{1 / q} .
\end{aligned}
$$

There are more known classes of multilinear operators related to the one given in Definition 2.1. The first remarkable one is given by the $p$-semi-integral multilinear operators. If $1 \leq$ $p<\infty$, we say that a multilinear operator $T: X_{1} \times \cdots \times X_{n} \rightarrow Y$ is $p$-semi-integral if for every set of finite sequences in the corresponding spaces we have

$$
\left(\sum_{j=1}^{M}\left\|T\left(x_{j}^{(1)}, \ldots, x_{j}^{(n)}\right)\right\|_{Y}^{p}\right)^{1 / p} \leq C \sup _{x_{1}^{*} \in B_{X_{1}^{*}, \ldots, x_{n}^{*} \in B_{X_{n}^{*}}}}\left(\sum_{j=1}^{M}\left|\left\langle x_{j}^{(1)}, x_{1}^{*}\right\rangle \cdots\left\langle x_{j}^{(n)}, x_{n}^{*}\right\rangle\right|^{p}\right)^{1 / p} .
$$

Thus, Definition 2.1 gives a "factorable version" of these operators with $q(q \neq p)$ on the left hand side of the inequality. We point out that there is a Pietsch's Domination Theorem which states that $T$ is $p$-semi-integral if and only if there exist a constant $C>0$ and a probablity measure $\mu$ on $B_{X_{1}^{*}} \times \cdots \times B_{X_{n}^{*}}$ such that for all $\left(x^{(1)}, \ldots, x^{(n)}\right) \in X_{1} \times \cdots \times X_{n}$,

$$
\left\|T\left(x^{(1)}, \ldots, x^{(n)}\right)\right\|_{Y} \leq C\left(\int_{B_{X_{1}^{*} \times \cdots \times B_{X_{n}^{*}}}}\left|\left\langle x^{(1)}, x_{1}^{*}\right\rangle \cdots\left\langle x^{(n)}, x_{n}^{*}\right\rangle\right|^{p} d \mu\right)^{1 / p} .
$$

We remark that $p$-semi-integral operators are closely related to the so called $\tau(p)$-summing (linear) operators, that were firstly introduced by Pietsch for $p=1$ in [29] and studied later in [23] for the general case. Although the definition was given for the case of linear operators, it involves the bilinear functional $T: X \times Y^{*} \rightarrow \mathbb{K}$,

$$
T\left(x, y^{*}\right)=\left\langle S(x), y^{*}\right\rangle, \quad\left(x, y^{*}\right) \in X \times Y^{*}
$$

associated to the linear operator $S: X \rightarrow Y$. Recently, more properties and applications of these operators have been studied in [34].

The second class of operators that is connected with our definition is the one of the factorable $p$-summing multilinear operators, that were in fact firstly given for polynomials. In [24] (see also the references therein) it is shown that this class satisfies a Pietsch-type Factorization Theorem through a $p$-summing operator composed with a canonical multilinear map. In this case, the canonical multilinear map is the product on the projective tensor product of the domain spaces.

The next class of operators to which we want to pay attention is the one of the so called integral multilinear operators, that were introduced and systematically analyzed in [35]. The 
particular case of Pietsch-integral linear operators has been explained above. A multilinear operator $T: X_{1} \times \cdots \times X_{n} \rightarrow Y$ is called Pietsch-integral ( $P$-integral) if there is a regular $Y$-valued Borel measure $G$ of bounded variation on $B_{X_{1}^{*}} \times \cdots \times B_{X_{n}^{*}}$ such that

$$
T\left(x^{(1)}, \ldots, x^{(n)}\right)=\int_{B_{X_{1}^{*} \times \cdots \times B_{X_{n}^{*}}}}\left\langle x^{(1)}, x_{1}^{*}\right\rangle \cdots\left\langle x^{(n)}, x_{n}^{*}\right\rangle d G\left(x_{1}^{*}, \ldots, x_{n}^{*}\right),
$$

for all $\left(x^{(1)}, \ldots, x^{(n)}\right) \in X_{1} \times \cdots \times X_{n}$. The following characterization is known (see [35, Proposition 2.6]): $T$ is $P$-integral if and only if the linearization $\widehat{T}$ of $T$ can be extended to the injective tensor product $X_{1} \widehat{\otimes}_{\varepsilon} \cdots \widehat{\otimes}_{\varepsilon} X_{n}$, and this linear extension is $P$-integral. This automatically implies a factorization through the $\varepsilon$ tensor product and a linear operator as

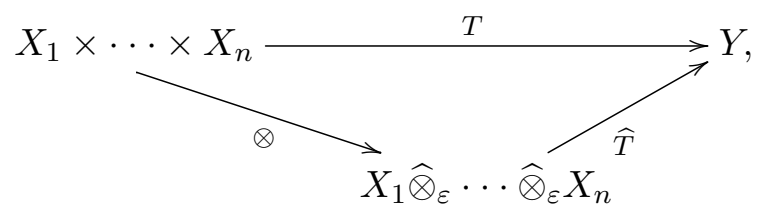

where $\widehat{T}$ is $P$-integral, and so factors itself through an $L^{1}$-space. This diagram is similar to the one we will get for our class of multilinear operators. The class which satisfy such a factorization but for the extended multilinear operator having values on $Y^{* *}$ was also studied in [35]. Finally, let us remark that another related space of linear operators acting in injective tensor products of Banach spaces was analyzed in [22]; see Theorem 2.4 below.

The notion that we have introduced is in a sense a mix of the definitions explained above. It is a "factorable" class, since it admits a factorization theorem starting with a canonical multilinear map (in this case a pointwise product) and the defining inequalities involve matrices of vectors. It is also of $\tau(p)$-summing type, since we have single products of functionals in the right hand side of the inequalities. Note also that we are interested in considering different exponents $q$ and $p$ in the definition, so it should be some sort of $\tau(p, q)$-summing operators of factorable type. Finally, let us point out that there are also other relevant relations with classical summing multi-ideals. For example, it is easy to prove by writing the inequalities in the definition for some particular class of matrices that all factorable $(q, 1)$-summing multilinear operators are multiple $(q, 1)$-summing. We are not interested in this class of operators in this paper and so we will not analyze the details of this relation.

We present an example showing that there exists a bounded bilinear functional which is not factorable $(q, p)$-summing.

Example 2.2. Consider the bilinear functional $\varphi: \ell^{2} \times \ell^{2} \rightarrow \mathbb{R}$ given by the canonical duality,

$$
\varphi\left(\left(\lambda_{j}\right),\left(\tau_{j}\right)\right):=\left\langle\left(\lambda_{j}\right),\left(\tau_{j}\right)\right\rangle=\sum_{j=1}^{\infty} \lambda_{i} \tau_{i}, \quad\left(\left(\lambda_{j}\right),\left(\tau_{j}\right)\right) \in \ell^{2} \times \ell^{2} .
$$

We claim that $\varphi$ is not factorable $(q, 1)$-summing. Indeed, take $M=1$, and the (one row) "matrix" $\left(s_{k}\right)$ of vectors in $\ell^{2}$, where $s_{k}=e_{k}$ for all $k \in \mathbb{N}$ and $e_{k}$ are the elements of the canonical basis of the space. Then, for each $n \in \mathbb{N}$, we get that

$$
\left|\sum_{k=1}^{n} \varphi\left(e_{k}, e_{k}\right)\right|=n
$$


Thus if $\varphi$ were factorable $(q, 1)$-summing, we would get that there is a constant $C>0$ such that

$$
\begin{aligned}
n=\left|\sum_{k=1}^{n} \varphi\left(e_{k}, e_{k}\right)\right| & \leq C \sup _{\left(\lambda_{j}\right) \in B_{\ell^{2}},\left(\tau_{j}\right) \in B_{\ell^{2}}}\left|\sum_{k=1}^{n}\left\langle e_{k},\left(\lambda_{j}\right)\right\rangle\left\langle e_{k},\left(\tau_{j}\right)\right\rangle\right| \\
& =C \sup _{\left(\lambda_{j}\right) \in B_{\ell^{2}},\left(\tau_{j}\right) \in B_{\ell^{2}}}\left|\sum_{k=1}^{n} \lambda_{k} \tau_{k}\right| \\
& \leq C \sup _{\left(\lambda_{j}\right) \in B_{\ell^{2}},\left(\tau_{j}\right) \in B_{\ell^{2}}}\left\|\left(\lambda_{j}\right)\right\|_{\ell^{2}}\left\|\left(\tau_{j}\right)\right\|_{\ell^{2}}=C .
\end{aligned}
$$

This example is relevant in the following sense: although it is given by the composition $\varphi=r \circ \odot$ of the pointwise multiplication $\odot: \ell^{2} \times \ell^{2} \rightarrow \ell^{1}$ and the linear map $r: \ell^{1} \rightarrow \mathbb{R}$, $r\left(\left(\lambda_{j}\right)\right)=\sum \lambda_{j}$, it is not factorable $(q, 1)$-summing. This direct relation with the pointwise multiplication maps holds only in the case of $C(K)$-spaces. However, our development may also give some non-trivial positive results for the case of bilinear maps acting in Banach function spaces and essentially defined by a pointwise product. We will show some of them in Subsection 2.3.

We will prove in this section that the notion introduced in Definition 2.1 is the one fitting with our aim of describing multilinear operators that satisfy a factorization involving a multiplication multilinear operator.

The following Proposition shows that the property of being factorable $(q, p)$-summing is preserved by composition with linear operators. Since the proof is obvious we skip it.

Proposition 2.3. Let $T: X_{1} \times \cdots \times X_{n} \rightarrow Y$ be a factorable $(q, p)$-summing operator, and let $A_{i}: Z_{i} \rightarrow X_{i}(1 \leq i \leq n), R: Y \rightarrow E$ be operators between Banach spaces. Then the $n$-linear operator $S: Z_{1} \times \cdots \times Z_{n} \rightarrow E$ given by $S=R \circ T \circ\left(A_{1}, \ldots, A_{n}\right)$ is also factorable $(q, p)$-summing.

The following result allows to relate the class of factorable $(q, p)$-summing operators with the norm in a space of continuous functions. It is our main characterization of this class of multilinear operators.

Let us introduce first some notation. Consider the compact set $B_{X_{1}^{*}} \times \cdots \times B_{X_{n}^{*}}$, where the weak* topologies are considered as usual. We denote by $\kappa_{X_{i}}$ the canonical embeddings $\kappa_{X_{i}}: X_{i} \rightarrow C\left(B_{X_{i}^{*}}\right)$ for each $1 \leq i \leq n$. If $S_{i}$ are nonempty sets and $f_{i}: S_{i} \rightarrow \mathbb{K}$ are functions for each $1 \leq i \leq n$, then $\odot$ denotes the pointwise product map defined by

$$
\odot\left(f_{1}, \ldots, f_{n}\right)\left(s_{1}, \ldots, s_{n}\right):=f_{1}\left(s_{1}\right) \cdots f_{n}\left(s_{n}\right), \quad\left(s_{1}, \ldots, s_{n}\right) \in S_{1} \times \cdots \times S_{n} .
$$

If $X_{1}, \ldots, X_{n}$ are Banach spaces, them using the pointwise product $\odot$, we define the multiplication operator $\circledast: X_{1} \times \cdots \times X_{n} \rightarrow C\left(B_{X_{1}^{*}} \times \cdots \times B_{X_{n}^{*}}\right)$ by

$$
\circledast\left(x^{(1)}, \ldots, x^{(n)}\right):=\odot\left(\kappa_{X_{1}}\left(x^{(1)}\right), \ldots, \kappa_{X_{n}}\left(x^{(n)}\right)\right), \quad\left(x^{(1)}, \ldots, x^{(n)}\right) \in X_{1} \times \cdots \times X_{n} .
$$

In what follows we will denote by $S$ the closure of the subspace of $C\left(B_{X_{1}^{*}} \times \cdots \times B_{X_{n}^{*}}\right)$ generated as the linear span of the range of the multilinear map $\circledast$.

We are ready to state the main result of this section, which gives a characterization of factorable $(q, p)$-summing multilinear operators in terms of factorization through the injective tensor product of Banach spaces. It relates directly our setting with the one in [22] where summing linear operators acting in injective tensor products are studied.

Theorem 2.4. Let $1 \leq p \leq q<\infty$. The following statements are equivalent for a Banach space valued $n$-linear operator $T: X_{1} \times \cdots \times X_{n} \rightarrow Y$.

(i) $T$ is factorable $(q, p)$-summing with a constant $C>0$. 
(ii) There is a $(q, p)$-summing linear operator $\widetilde{T}: X_{1} \widehat{\otimes}_{\varepsilon} \cdots \widehat{\otimes}_{\varepsilon} X_{n} \rightarrow Y$ with $\pi_{q, p}(\widetilde{T}) \leq C$ such that $T$ admits the factorization:

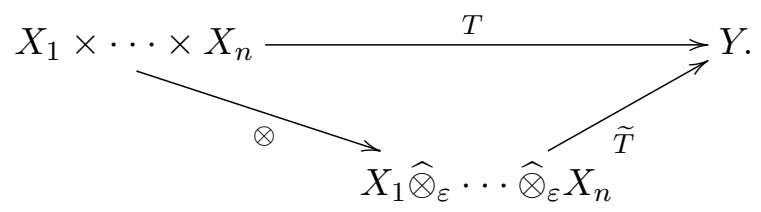

(iii) There exists a constant $C>0$ such that for each positive integers $M, N$ and all $M \times N$ matrices $\left(x_{j k}^{(1)}\right)$ in $X_{1}, \ldots,\left(x_{j k}^{(n)}\right)$ in $X_{n}$, the following inequality holds:

$$
\begin{aligned}
& \left(\sum_{j=1}^{M}\left\|\sum_{k=1}^{N} T\left(x_{j k}^{(1)}, \ldots, x_{j k}^{(n)}\right)\right\|_{Y}^{q}\right)^{1 / q} \\
& \leq C\left\|\sum_{j=1}^{M}\left|\sum_{k=1}^{N} \odot\left(\kappa_{X_{1}}\left(x_{j k}^{(1)}\right), \ldots, \kappa_{X_{n}}\left(x_{j k}^{(n)}\right)\right)\right|^{p}\right\|_{C\left(B_{X_{1}^{*}} \times \cdots \times B_{X_{n}^{*}}\right)}^{1 / p} .
\end{aligned}
$$

(iv) There is a $(q, p)$-summing linear operator $\widetilde{T}: S \rightarrow Y$ with $\pi_{q, p}(\widetilde{T}) \leq C$ such that $T$ admits the following factorization through a subspace $S$ of $C\left(B_{X_{1}^{*}} \times \cdots \times B_{X_{n}^{*}}\right)$ :



Proof. (i) $\Leftrightarrow$ (ii). Let us show first that if $T$ is a factorable $(q, p)$-summing $n$-linear operator $T: X_{1} \times \cdots \times X_{n} \rightarrow Y$, then $T$ admits a factorization:

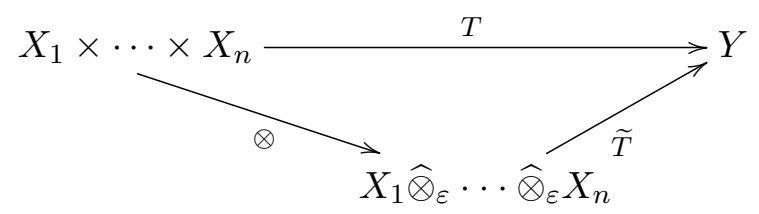

where $\otimes$ is the universal $n$-linear mapping and $\widetilde{T}$ is a bounded linear operator.

If we take $N=1$, then our hypothesis implies that for every finite sequence of elements $\left(\left(x_{k}^{(1)}, \ldots, x_{k}^{(n)}\right)\right)_{k=1}^{m}$ in $X_{1} \times \cdots \times X_{n}$, we have



$$
\begin{aligned}
& =C^{p} \varepsilon\left(\sum_{k=1}^{m} x_{k}^{(1)} \otimes \cdots \otimes x_{k}^{(n)}\right)^{p} .
\end{aligned}
$$

Since the subspace spanned by the $x_{1} \otimes \cdots \otimes x_{n}$ is dense in the injective tensor product, the above estimate allows us to define a unique bounded operator $\widetilde{T}: X_{1} \widehat{\otimes}_{\varepsilon} \cdots \widehat{\otimes}_{\varepsilon} X_{n} \rightarrow Y$ satisfying

$$
\widetilde{T}\left(x_{1} \otimes \cdots \otimes x_{n}\right)=T\left(x_{1}, \ldots, x_{n}\right), \quad\left(x_{1}, \ldots, x_{n}\right) \in X_{1} \times \cdots \times X_{n} .
$$

We claim that the operator $\widetilde{T}$ is $(q, p)$-summing. To see this, we take a sequence of tensors $\left(\sum_{k=1}^{N} x_{j k}^{(1)} \otimes \cdots \otimes x_{j k}^{(n)}\right)_{j=1}^{M}$. Recall that the dual space $\left(X_{1} \otimes_{\varepsilon} \cdots \otimes_{\varepsilon} X_{n}\right)^{*}$ is isometrically 
isomorphic to the space of all integral $n$-linear functionals. Every $\varphi \in\left(X_{1} \otimes_{\varepsilon} \cdots \otimes_{\varepsilon} X_{n}\right)^{*}$ is represented by an integral formula given by

$$
\left\langle x^{(1)} \otimes \cdots \otimes x^{(n)}, \varphi\right\rangle=\int_{B_{X_{1}^{*}} \times \cdots \times B_{X_{n}^{*}}}\left\langle x^{(1)}, x_{1}^{*}\right\rangle \cdots\left\langle x^{(n)}, x_{n}^{*}\right\rangle d \mu,
$$

where $\mu$ is a positive regular Borel measure on the compact set $B_{X_{1}^{*}} \times \cdots \times B_{X_{n}^{*}}$ endowed with the product of the weak* topologies such that $\|\varphi\|=\mu\left(B_{X_{1}^{*}} \times \cdots \times B_{X_{n}^{*}}\right)$ (see, e.g., [7, Th.4.6] for the bilinear case; see also [35, Sec.2] for the general vector-valued case). Below we write $\mathcal{M}$ in the following computations for the probability measures like these ones. We have that with $C=\mu\left(B_{X_{1}^{*}} \times \cdots \times B_{X_{n}^{*}}\right)$

$$
\begin{aligned}
\sup _{\varphi \in B_{\left(X_{1} \otimes_{\varepsilon} \cdots \otimes_{\varepsilon} X_{n}\right)^{*}}} & \left(\sum_{j=1}^{M}\left|\left\langle\sum_{k=1}^{N} x_{j k}^{(1)} \otimes \cdots \otimes x_{j k}^{(n)}, \varphi\right\rangle\right|^{p}\right)^{1 / p} \\
& \left.=\left.\sup _{\mu \in \mathcal{M}}\left(\sum_{j=1}^{M} \mid \int_{B_{X_{1}^{*}} \times \cdots \times B_{X_{n}^{*}}} \sum_{k=1}^{N}\left\langle x_{j k}^{(1)}, x_{1}^{*}\right\rangle \cdots\left\langle x_{j k}^{(n)}, x_{n}^{*}\right\rangle\right) d \mu\right|^{p}\right)^{1 / p} \\
& =\sup _{\mu \in \mathcal{M}} \sup _{\left(\lambda_{j}\right) \in B_{\ell p^{\prime}}} \int_{B_{X_{1}^{*}} \times \cdots \times B_{X_{n}^{*}}}\left(\sum_{j=1}^{M} \lambda_{j}\left(\sum_{k=1}^{N}\left\langle x_{j k}^{(1)}, x_{1}^{*}\right\rangle \cdots\left\langle x_{j k}^{(n)}, x_{n}^{*}\right\rangle\right)\right) d \mu \\
& \leq \sup _{x_{1}^{*} \in B_{X_{1}^{*}}, \ldots, x_{n}^{*} \in B_{X_{n}^{*}}}\left(\sum_{j=1}^{M}\left|\sum_{k=1}^{N}\left\langle x_{j k}^{(1)}, x_{1}^{*}\right\rangle \cdots\left\langle x_{j k}^{(n)}, x_{n}^{*}\right\rangle\right|^{p}\right)^{1 / p} .
\end{aligned}
$$

Since the supremum in this expression is in fact a maximum, we conclude that there is Dirac's delta $\delta_{x_{1}^{*}, \ldots, x_{n}^{*}} \in \mathcal{M}$ of an element $\left(x_{1}^{*}, \ldots, x_{n}^{*}\right) \in B_{X_{1}^{*}} \times \cdots \times B_{X_{n}^{*}}$ such that

$$
\begin{aligned}
\sup _{\varphi \in B_{\left(X_{1} \otimes_{\varepsilon} \cdots \otimes_{\varepsilon} X_{n}\right)^{*}}} & \left(\sum_{j=1}^{M}\left|\left\langle\sum_{k=1}^{N} x_{j k}^{(1)} \otimes \cdots \otimes x_{j k}^{(n)}, \varphi\right\rangle\right|^{p}\right)^{1 / p} \\
& =\left(\sum_{j=1}^{M}\left|\int_{B_{X_{1}^{*} \times \cdots \times B_{X_{n}^{*}}}}\left(\sum_{k=1}^{N}\left\langle x_{j k}^{(1)}, x_{1}^{*}\right\rangle \cdots\left\langle x_{j k}^{(n)}, x_{n}^{*}\right\rangle\right) d \delta_{x_{1}^{*}, \ldots, x_{n}^{*}}\right|^{p}\right)^{1 / p} .
\end{aligned}
$$

This shows that the inequality appearing in the definition of factorable $(q, p)$-summing for $T$ and the one in the definition of $\widetilde{T}$ to be $(q, p)$-summing coincide. In consequence this proves that the conditions (i) and (ii) are equivalent.

(i) $\Leftrightarrow$ (iii). Take $x^{(1)} \in X_{1}, \ldots, x^{(n)} \in X_{n}$. The definition of the norm of a $C(K)$ space gives the equality

$$
\begin{aligned}
\sup _{x_{1}^{*} \in B_{X_{1}^{*}, \ldots, x_{n}^{*} \in B_{X_{n}^{*}}}} \sum_{j=1}^{M}\left|\sum_{k=1}^{N}\left\langle x_{j k}^{(1)}, x_{1}^{*}\right\rangle \cdots\left\langle x_{j k}^{(n)}, x_{n}^{*}\right\rangle\right|^{p} \\
=\left\|\sum_{j=1}^{M}\left|\sum_{k=1}^{N} \odot\left(\kappa_{X_{1}}\left(x_{j k}^{(1)}\right), \ldots, \kappa_{X_{n}}\left(x_{j k}^{(n)}\right)\right)\right|^{p}\right\|_{C\left(B_{X_{1}^{*}} \times \cdots \times B_{X_{n}^{*}}\right)}
\end{aligned}
$$

This yields the equivalence between (i) and (iii).

(iii) $\Rightarrow$ (iv). Consider the subspace $S_{0}$ of $C\left(B_{X_{1}^{*}} \times \cdots \times B_{X_{n}^{*}}\right)$ generated by all the linear combinations of the functions $\odot\left(\kappa_{X_{1}}\left(x^{(1)}\right), \ldots, \kappa_{X_{n}}\left(x^{(n)}\right)\right)$, where $x^{(1)} \in X_{1}, \ldots, x^{(n)} \in X_{n}$. This is described as finite sums of products of functions $\sum_{k=1}^{N}\left\langle x_{k}^{(1)}, \cdot\right\rangle \cdots\left\langle x_{k}^{(n)}, \cdot\right\rangle$ acting in the compact set $B_{X_{1}^{*}} \times \cdots \times B_{X_{n}^{*}}$; notice that there is no need of writing explicitly the product by scalars in each summand, since we can include them, for example, in the first coordinate. 
Let $S$ be the closure of $S_{0}$ in $C\left(B_{X_{1}^{*}} \times \cdots \times B_{X_{n}^{*}}\right)$. We will use the well known isometrical duality formulas:

$$
S^{*} \simeq C\left(B_{X_{1}^{*}} \times \cdots \times B_{X_{n}^{*}}\right)^{*} / S^{\perp}, \quad C\left(B_{X_{1}^{*}} \times \cdots \times B_{X_{n}^{*}}\right)^{*} \simeq \mathcal{M}\left(B_{X_{1}^{*}} \times \cdots \times B_{X_{n}^{*}}\right) .
$$

Since the set of all functions represented by $f_{j}=\sum_{k=1}^{N}\left\langle x_{j k}^{(1)}, \cdot\right\rangle \cdots\left\langle x_{j k}^{(n)}, \cdot\right\rangle$ is dense in the space $S$, we can define the operator $\widetilde{T}: S \rightarrow Y$ by

$$
\widetilde{T}\left(f_{j}\right):=\sum_{k=1}^{N} T\left(x_{j k}^{(1)}, \ldots, x_{j k}^{(n)}\right) .
$$

Note that $\widetilde{T}$ is well defined by the injectivity of the map $\circledast$. Next, observe that the inequality in (ii) implies that $\widetilde{T}$ is a continuous operator from $S$ into $Y$. Clearly, we have the factorization $T=\widetilde{T} \circ \circledast$.

On the other hand, we have

$$
\begin{aligned}
& \left\|\sum_{j=1}^{M}\left|\sum_{k=1}^{N}\left\langle x_{j k}^{(1)}, \cdot\right\rangle \cdots\left\langle x_{j k}^{(n)}, \cdot\right\rangle\right|^{p}\right\|_{C\left(B_{X_{1}^{*}} \times \cdots \times B_{X_{n}^{*}}\right)}
\end{aligned}
$$

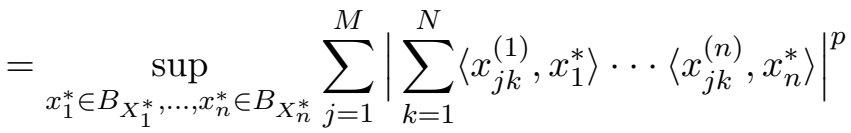

$$
\begin{aligned}
& =\sup _{x_{1}^{*} \in B_{X_{1}^{*}}, \ldots, x_{n}^{*} \in B_{X_{n}^{*}}} \sum_{j=1}^{M}\left|\left\langle\sum_{k=1}^{N}\left\langle x_{j k}^{(1)}, \cdot\right\rangle \cdots\left\langle x_{j k}^{(n)}, \cdot\right\rangle, x_{1}^{*} \otimes \cdots \otimes x_{n}^{*}\right\rangle\right|^{p} \\
& \leq \sup _{\varphi \in B_{\mathcal{M}\left(B_{X_{1}^{*}} \times \cdots \times B_{\left.X_{n}^{*}\right)}\right.}} \sum_{j=1}^{M}\left|\left\langle\sum_{k=1}^{N}\left\langle x_{j k}^{(1)}, \cdot\right\rangle \cdots\left\langle x_{j k}^{(n)}, \cdot\right\rangle, \varphi\right\rangle\right|^{p} \\
& =\sup _{\phi \in B_{\mathcal{M}\left(B_{X_{1}^{*}} \times \cdots \times B_{\left.X_{n}^{*}\right) / S^{\perp}}\right.}} \sum_{j=1}^{M}\left|\left\langle\sum_{k=1}^{N}\left\langle x_{j k}^{(1)}, \cdot\right\rangle \cdots\left\langle x_{j k}^{(n)}, \cdot\right\rangle, \phi\right\rangle\right|^{p} \\
& =\sup _{\phi \in B_{S^{*}}} \sum_{j=1}^{M}\left|\left\langle\sum_{k=1}^{N}\left\langle x_{j k}^{(1)}, \cdot\right\rangle \cdots\left\langle x_{j k}^{(n)}, \cdot\right\rangle, \phi\right\rangle\right|^{p} \text {. }
\end{aligned}
$$

Combining these computations with the inequality in condition (ii) for the factorable $(q, p)$ summing operators applied for the functions $f_{j}=\sum_{k=1}^{N}\left\langle x_{j k}^{(1)}, \cdot\right\rangle \cdots\left\langle x_{j k}^{(n)}, \cdot\right\rangle$ for each $1 \leq j \leq M$, we get that

$$
\left(\sum_{j=1}^{M}\left\|\widetilde{T}\left(f_{j}\right)\right\|_{Y}^{q}\right)^{1 / q} \leq C \sup _{\phi \in B_{S^{*}}}\left(\sum_{k=1}^{M}\left|\left\langle f_{j}, \phi\right\rangle\right|^{p}\right)^{1 / p} .
$$

Thus $\widetilde{T}$ is $(q, p)$-summing and so the claim is established. This proves (iii) $\Rightarrow$ (iv). 
(iv) $\Rightarrow$ (iii). Assume that $T$ admits a factorization $T=\widetilde{T} \circ \circledast$ as shown in (iii). Consider $M \times N$ matrices $\left(x_{j k}^{(1)}\right), \ldots,\left(x_{j k}^{(n)}\right)$ in $X_{1}, \ldots, X_{n}$, respectively. Then we have

$$
\begin{aligned}
& \left(\sum_{j=1}^{M}\left\|\sum_{k=1}^{N} T\left(x_{j k}^{(1)}, \ldots, x_{j k}^{(n)}\right)\right\|_{Y}^{q}\right)^{1 / q}=\left(\sum_{j=1}^{M}\left\|\sum_{k=1}^{N} \widetilde{T}\left(\circledast\left(x_{j k}^{(1)}, \ldots, x_{j k}^{(n)}\right)\right)\right\|_{Y}^{q}\right)^{1 / q} \\
& \leq \pi_{q, p}(\widetilde{T}) \sup _{\phi \in B_{S^{*}}}\left(\sum_{j=1}^{M}\left|\left\langle\sum_{k=1}^{N}\left\langle x_{j k}^{(1)}, \cdot\right\rangle \cdots\left\langle x_{j k}^{(n)}, \cdot\right\rangle, \phi\right\rangle\right|^{p}\right)^{1 / p} \\
& =\pi_{q, p}(\widetilde{T}) \sup _{\left(\lambda_{j}\right) \in B_{\ell p^{\prime}}}\left\|\sum_{j=1}^{M} \lambda_{j}\left(\sum_{k=1}^{N}\left\langle x_{j k}^{(1)}, \cdot\right\rangle \cdots\left\langle x_{j k}^{(n)}, \cdot\right\rangle\right)\right\|_{C\left(B_{X_{1}^{*}} \times \cdots \times B_{X_{n}^{*}}\right)} \\
& \leq \pi_{q, p}(\widetilde{T})\left\|\sup _{\left(\lambda_{j}\right) \in B_{\ell p^{\prime}}}\left(\sum_{j=1}^{M} \lambda_{j}\left(\sum_{k=1}^{N}\left\langle x_{j k}^{(1)}, \cdot\right\rangle \cdots\left\langle x_{j k}^{(n)}, \cdot\right\rangle\right)\right)\right\|_{C\left(B_{X_{1}^{*}} \times \cdots \times B_{X_{n}^{*}}\right)} \\
& \leq \pi_{q, p}(\widetilde{T}) \sup _{x_{1}^{*} \in B_{X_{1}^{*}, \ldots, x_{n}^{*} \in B_{X_{n}^{*}}}}\left(\sum_{j=1}^{M}\left|\sum_{k=1}^{N}\left\langle x_{j k}^{(1)}, x_{1}^{*}\right\rangle \cdots\left\langle x_{j k}^{(n)}, x_{n}^{*}\right\rangle\right|^{p}\right)^{1 / p} .
\end{aligned}
$$

This proves (iii) $\Rightarrow$ (i) and completes the proof.

To conclude we remark here that the computations given in the proof allows to assure also that the infimum of all the constants appearing $C$ in the definition of factorable $(q, p)$ summing coincides with $\pi_{q, p}(\widetilde{T})$ for the operator $\widetilde{T}$ appearing in the factorization of $T$.

We will show in the following subsections of this section some applications to bilinear operators factoring through pointwise multiplication operators which appear in problems related to some classical extension theorems.

2.1. The first variant of Pisier's Theorem. As in the case of linear $p$-summing operators, the factorization theorems that are obtained for the case of $C(K)$ as domain spaces are special. The reason is that in this case, the operator is already acting in $C(K)$-spaces, and is not necessary to embed the domain $X$ in the space of continuous functions $C\left(B_{X^{*}}\right)$. We will show in this section how we can provide a factorization of the multilinear operator using the specific elements appearing in this space. In particular, the pointwise product $f^{(1)} \cdots f^{(n)}$ of the original functions $f^{(1)} \in C\left(K_{1}\right), \ldots, f^{(n)} \in C\left(K_{n}\right)$ will play a key role.

One of our aims is to investigate the class of multilinear operators on the product of $C(K)$-spaces for which a multilinear variant of Pisier's result is true. The indicated topic of this section is inspired by the remarkable factorization theorem due to Pisier [31], which asserts that an operator $T$ from a $C(K)$-space to a Banach space $Y$ is $(q, p)$-summing with $1 \leq p<q<\infty$ if and only if there is a probability Borel measure $\mu$ on $K$ such that $T$ factors as follows:

$$
T: C(K) \stackrel{j}{\longrightarrow} L_{q, 1}(\mu) \stackrel{S}{\longrightarrow} Y
$$

where $j$ is the continuous inclusion map. Here $L_{q, 1}(\mu)$ is the Lorentz space on the measure space $(K, \mathcal{B}(K), \mu)$ equipped with the norm

$$
\|f\|:=\int_{0}^{1} f^{*}(t) t^{1 / q-1} d t
$$

where $\mathcal{B}(K)$ is the $\sigma$-algebra of the Borel sets in $K$ and $f^{*}(t)=\inf \{s>0 ; \mu(\{|f|>s\}) \leq t\}$, $t \in[0,1]$ is the decreasing rearrangement of $|f|$. Notice that Pisier's Theorem is a cornerstone in the theory of $(q, p)$-concave operators, which is deeply connected with the linear theory of $(q, p)$-summing operators (see, e.g., [10, pp. 326-345]). 
Now we are ready to state the multilinear version of Pisier's Factorization Theorem. For simplicity of presentation we state only a bilinear version; the proof of the multilinear version is similar via Theorem 2.4(iii).

Theorem 2.5. Let $1 \leq p<q<\infty$. The following statements are equivalent for a Banach space valued bilinear map $T: C\left(K_{1}\right) \times C\left(K_{2}\right) \rightarrow Y$.

(i) $T$ is factorable $(q, p)$-summing.

(ii) For each positive integers $M, N$ and all $M \times N$ matrices $\left(f_{j k}\right)$ and $\left(g_{j k}\right)$ in $C\left(K_{1}\right)$ and $C\left(K_{2}\right)$, respectively, the following inequality holds

$$
\left(\sum_{j=1}^{M}\left\|\sum_{k=1}^{N} T\left(f_{j k}, g_{j k}\right)\right\|^{q}\right)^{1 / q} \leq C\left\|\left(\sum_{j=1}^{M}\left|\sum_{k=1}^{N} \odot\left(f_{j k}, g_{j k}\right)\right|^{p}\right)^{1 / p}\right\|_{C\left(K_{1} \times K_{2}\right)} .
$$

(iii) There is a probability Borel measure $\mu$ on $K_{1} \times K_{2}$ such that $T$ admits a factorization:

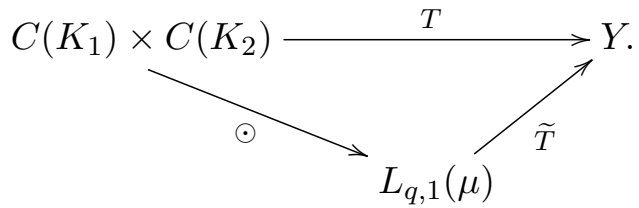

Proof. Since the set of all evaluation functionals forms a norming set in any $C(K)$-space, it easily follows that for all $M \times N$ matrices $\left(f_{j k}\right)$ and $\left(g_{j k}\right)$ in $C\left(K_{1}\right)$ and $C\left(K_{2}\right)$, respectively,

$$
\begin{aligned}
\sup _{\varphi \in B_{C\left(K_{1}\right)^{*}}, \psi \in B_{C\left(K_{2}\right)^{*}}} & \left(\sum_{j=1}^{M}\left|\sum_{k=1}^{N}\left\langle f_{j k}, \varphi\right\rangle\left\langle g_{j k}, \psi\right\rangle\right|^{p}\right)^{1 / p} \\
& =\left\|\left(\sum_{j=1}^{M}\left|\sum_{k=1}^{N} \odot\left(f_{j k}, g_{j k}\right)\right|^{p}\right)^{1 / p}\right\|_{C\left(K_{1} \times K_{2}\right)} .
\end{aligned}
$$

Thus, by the natural identification of $C\left(K_{1}\right) \widehat{\otimes}_{\varepsilon} C\left(K_{2}\right)$ with $C\left(K_{1} \times K_{2}\right)$, it follows that (i) is equivalent to (ii).

(ii) $\Rightarrow$ (iii). Applying Theorem 2.4(ii), we conclude that the operator $T$ factors through $C\left(K_{1} \times K_{2}\right)$. Denote by $T_{0}$ the linear operator $\widetilde{T}$ which appears in the factorization Theorem 2.4(ii), and note that it satisfies the inequality

$$
\left(\sum_{j=1}^{M}\left\|T_{0}\left(\sum_{k=1}^{N} \odot\left(f_{j k}, g_{j k}\right)\right)\right\|^{q}\right)^{1 / q} \leq C\left\|\left(\sum_{j=1}^{M}\left|\sum_{k=1}^{N} \odot\left(f_{j k}, g_{j k}\right)\right|^{p}\right)^{1 / p}\right\|_{C\left(K_{1} \times K_{2}\right)}
$$

for all $M \times N$ matrices $\left(f_{j k}\right)$ and $\left(g_{j k}\right)$ in $C\left(K_{1}\right)$ and $C\left(K_{2}\right)$, respectively. Since the set of functions of the form $\sum_{j=1}^{M} \odot\left(f_{j}, g_{j}\right)$ is dense in $C\left(K_{1} \times K_{2}\right)$, the above inequality holds for all functions in $C\left(K_{1} \times K_{2}\right)$. This yields that for all $h_{j} \in C\left(K_{1} \times K_{2}\right), 1 \leq j \leq n$,

$$
\left(\sum_{j=1}^{n}\left\|T_{0}\left(h_{j}\right)\right\|_{Y}^{q}\right)^{1 / q} \leq C\left\|\left(\sum_{j=1}^{n}\left|h_{j}\right|^{p}\right)^{1 / p}\right\|_{C\left(K_{1} \times K_{2}\right)}
$$

and so $T_{0}: C\left(K_{1} \times K_{2}\right) \rightarrow Y$ is $(q, 1)$-summing. Then Pisier's Theorem [31] (see also, [10, Theorem 10.9]) gives the desired factorization by taking $\widetilde{T}=T_{0} \circ i$, where $i: C\left(K_{1} \times K_{2}\right) \hookrightarrow$ $L_{q, 1}(\mu)$ is the natural identification map.

(iii) $\Rightarrow$ (ii). Suppose that $T$ factors as it is shown in (iii). Since $\mu$ is a probability Borel measure on $K_{1} \times K_{2}$, the canonical inclusion map $i: C\left(K_{1} \times K_{2}\right) \rightarrow L_{q, 1}(\mu)$ is continuous; in fact, it is easy to check that it is $(q, 1)$-summing. Since via a natural identification $C\left(K_{1} \times\right.$ 
$\left.K_{2}\right)$ is the injective tensor product of $C\left(K_{1}\right)$ and $C\left(K_{2}\right)$, it follows that the bilinear map $\odot: C\left(K_{1}\right) \times C\left(K_{2}\right) \rightarrow C\left(K_{1} \times K_{2}\right)$ is bounded. To summarize, we have that the bilinear operator $T$ admits a factorization:

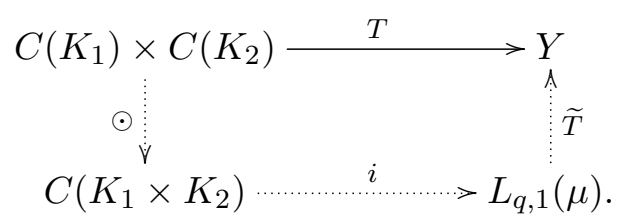

Now, applying the linear Pisier's Theorem we conclude that for all $M \times N$ matrices $\left(f_{j k}\right)$ and $\left(g_{j k}\right)$ in $C\left(K_{1}\right)$ and $C\left(K_{2}\right)$, respectively,

$$
\left(\sum_{j=1}^{M}\left\|\sum_{k=1}^{N} T\left(f_{j k}, g_{j k}\right)\right\|^{q}\right)^{1 / q} \leq C\left\|\left(\sum_{j=1}^{M}\left|\sum_{k=1}^{N} \odot\left(f_{j k}, g_{j k}\right)\right|^{p}\right)^{1 / p}\right\|_{C\left(K_{1} \times K_{2}\right)}
$$

and this proves (ii).

Note that we have actually proved that a bilinear operator $T: C\left(K_{1}\right) \times C\left(K_{2}\right) \rightarrow Y$ is factorable $(q, p)$-summing if and only if it factors as



where $\mu$ is a probability Borel measure on $K_{1} \times K_{2}$. That is, factorization through the injective tensor product is implicitly guarantied. Other relevant fact regarding this factorization is connected with the so called Fremlin tensor product of Banach lattices (see [11]). It is wellknown that for the case of products of $C(K)$-spaces, the injective tensor product an the Fremlin tensor product coincides (see [11, Corollaries 3E]). We will develop this particular point in the last section of the paper.

2.2. Grothendieck's Theorem for factorable $(q, p)$-summing operators. We recall that Grothendieck's Factorization Theorem states that if $K_{1}$ and $K_{2}$ are locally compact Hausdorff spaces and $U$ is a bounded bilinear functional on $C_{0}\left(K_{1}\right) \times C_{0}\left(K_{2}\right)$, then there exist probability Borel measures $\mu_{1}, \mu_{2}$, on $K_{1}, K_{2}$, respectively, such that for all $f_{1} \in C_{0}\left(K_{1}\right)$, $f_{2} \in C\left(K_{2}\right)$,

$$
\left|U\left(f_{1}, f_{2}\right)\right| \leq \kappa_{G}\|U\|\left(\int_{K_{1}}\left|f_{1}\right|^{2} d \mu_{1}\right)^{1 / 2}\left(\int_{K_{2}}\left|f_{2}\right|^{2} d \mu_{2}\right)^{1 / 2},
$$

where $\kappa_{G}$ is universal constant.

We refer to [32] where a nice presentation of this classical topic is given. A multilinear variant of Grothendieck's Theorem is known (see [5, Theorem 3.2]). In this section we will show that all factorable $(q, p)$-summing bilinear (vector valued) operators satisfy Grothendieck's Theorem, and that this can be translated to a factorization scheme.

In what follows we assume that $K_{1}$ and $K_{2}$ are given locally compact Hausdorff spaces. Let $T: C_{0}\left(K_{1}\right) \times C_{0}\left(K_{2}\right) \rightarrow Y$ be a factorable $(q, p)$-summing operator. Then it defines two operators $T_{1}: C_{0}\left(K_{1}\right) \rightarrow L\left(C_{0}\left(K_{2}\right), Y\right)$ and $T_{2}: C_{0}\left(K_{2}\right) \rightarrow L\left(C_{0}\left(K_{1}\right), Y\right)$ in the standard way, for given $f \in C_{0}\left(K_{1}\right)$ and $g \in C_{0}\left(K_{2}\right)$,

$$
T_{1}(f)=T(f, \cdot): C_{0}\left(K_{2}\right) \rightarrow Y, \quad T_{2}(g)=T(\cdot, g): C_{0}\left(K_{1}\right) \rightarrow Y .
$$

We state the following technical result. 
Proposition 2.6. Let $T: C_{0}\left(K_{1}\right) \times C_{0}\left(K_{2}\right) \rightarrow Y$ be a factorable $(q, p)$-summing bilinear operator. Then the associated linear operators $T_{1}$ and $T_{2}$ are $(q, p)$-summing.

Proof. Let $\left(f_{j}\right)_{j=1}^{M}$ be a given sequence in $C_{0}\left(K_{1}\right)$. Then for a given $\varepsilon>0$, we find $\left(g_{j}\right)_{j=1}^{M}$ in the unit ball of $C_{0}\left(K_{2}\right)$ such that

$$
\left\|T_{1}\left(f_{j}\right)\right\|_{L\left(C_{0}\left(K_{2}\right), Y\right)} \leq(1+\varepsilon)\left\|T_{1}\left(f_{j}\right) g_{j}\right\|_{Y}=\left\|T\left(f_{j}, g_{j}\right)\right\|_{Y} .
$$

If $T$ is factorable $(q, p)$-summing with a constant $C$, we obtain (by considering only $M \times N$ matrices with $N=1$ )

$$
\begin{aligned}
\left(\sum_{j=1}^{M}\left\|T_{1}\left(f_{j}\right)\right\|_{L\left(C_{0}\left(K_{2}\right), Y\right)}^{q}\right)^{1 / q} & \leq(1+\varepsilon)\left(\sum_{j=1}^{M}\left\|T\left(f_{j}, g_{j}\right)\right\|_{Y}^{q}\right)^{1 / q} \\
& \leq C(1+\varepsilon)\left\|\left(\sum_{j=1}^{M}\left|\odot\left(f_{j}, g_{j}\right)\right|^{p}\right)^{1 / p}\right\|_{C_{0}\left(K_{1}\right)} \\
& \leq C(1+\varepsilon)\left\|\left(\sum_{j=1}^{M}\left|f_{j}\right|^{p}\right)^{1 / p}\right\|_{C_{0}\left(K_{1}\right)}
\end{aligned}
$$

Since $\varepsilon>0$ was arbitrary, we get that $T_{1}$ is $(q, p)$-summing with $\pi_{q, p}\left(T_{1}\right) \leq C$. Similar arguments prove the result for $T_{2}$.

Proposition 2.7. Let $1 \leq p \leq q<\infty$ and let $T: C_{0}\left(K_{1}\right) \times C_{0}\left(K_{2}\right) \rightarrow Y$ be a factorable $(q, p)$-summing bilinear operator. Then the following statements hold:

(i) There exists a constant $C>0$ such that for every sequence $\left(f_{j}\right)_{j=1}^{M}$ in $C_{0}\left(K_{1}\right)$ and $\left(g_{j}\right)_{j=1}^{M}$ in $C_{0}\left(K_{2}\right)$, we have

$$
\left(\sum_{j=1}^{M}\left\|T\left(f_{j}, g_{j}\right)\right\|_{Y}^{q}\right)^{1 / q} \leq C\left\|\left(\sum_{j=1}^{M}\left|f_{j}\right|^{2}\right)^{1 / 2}\right\|_{C_{0}\left(K_{1}\right)}\left\|\left(\sum_{j=1}^{M}\left|f_{j}\right|^{2}\right)^{1 / 2}\right\|_{C_{0}\left(K_{2}\right)} .
$$

(ii) There exists a constant $C>0$ such that for every sequence of functions $\left(f_{j}\right)_{j=1}^{M}$ in $C_{0}\left(K_{1}\right)$ and $\left(g_{j}\right)_{j=1}^{M}$ in $C_{0}\left(K_{2}\right)$, we have

$$
\left\|\sum_{j=1}^{M} T\left(f_{j}, g_{j}\right)\right\|_{Y} \leq C\left\|\left(\sum_{j=1}^{M}\left|f_{j}\right|^{2}\right)^{1 / 2}\right\|_{C_{0}\left(K_{1}\right)}\left\|\left(\sum_{j=1}^{M}\left|f_{j}\right|^{2}\right)^{1 / 2}\right\|_{C_{0}\left(K_{2}\right)} .
$$

Proof. Our hypothesis implies that there exists a constant $C>0$ such that for all $M \times N$ matrices $\left(f_{k j}\right)$ and $\left(g_{k j}\right)$ in $C_{0}\left(K_{1}\right)$ and $C_{0}\left(K_{2}\right)$, respectively, we have

$$
\begin{aligned}
& \left(\sum_{j=1}^{M}\left\|\sum_{k=1}^{N} T\left(f_{k j}, g_{k j}\right)\right\|_{Y}^{q}\right)^{1 / q} \leq C\left\|\left(\sum_{j=1}^{M}\left(\sum_{k=1}^{N}\left|f_{k j}\right| \cdot\left|g_{k j}\right|\right)^{p}\right)^{1 / p}\right\|_{C_{0}\left(K_{1} \times K_{2}\right)} \\
& \leq C\left\|\sum_{j=1}^{M} \sum_{k=1}^{N}\left|f_{k j}\right| \cdot\left|g_{k j}\right|\right\|_{C_{0}\left(K_{1} \times K_{2}\right)}=C\left\|\sum_{k=1}^{N} \sum_{j=1}^{M}\left|f_{k j}\right| \cdot\left|g_{k j}\right|\right\|_{C_{0}\left(K_{1} \times K_{2}\right)} \\
& \leq C \sum_{k=1}^{N}\left\|\sum_{j=1}^{M}\left|f_{k j}\right| \cdot\left|g_{k j}\right|\right\|_{C_{0}\left(K_{1} \times K_{2}\right)} \leq C \sum_{k=1}^{N}\left\|\left(\sum_{j=1}^{M}\left|f_{k j}\right|^{2}\right)^{1 / 2}\left(\sum_{j=1}^{M}\left|g_{k j}\right|^{2}\right)^{1 / 2}\right\| \|_{C_{0}\left(K_{1} \times K_{2}\right)} \\
& \leq C \sum_{k=1}^{N}\left\|\left(\sum_{j=1}^{M}\left|f_{k j}\right|^{2}\right)^{1 / 2}\right\|_{C_{0}\left(K_{1}\right)}\left\|\left(\sum_{j=1}^{M}\left|g_{k j}\right|^{2}\right)^{1 / 2}\right\|_{C_{0}\left(K_{2}\right)} .
\end{aligned}
$$


Applying the above in the case $N=1$, we get that for any finite sequences $\left(f_{j}\right)_{j=1}^{M}$ and $\left(g_{j}\right)_{j=1}^{M}$ in $C_{0}\left(K_{1}\right)$ and $C_{0}\left(K_{2}\right)$, respectively we obtain

$$
\left(\sum_{j=1}^{M}\left\|T\left(f_{j}, g_{j}\right)\right\|_{Y}^{q}\right)^{1 / q} \leq C\left\|\left(\sum_{j=1}^{M}\left|f_{j}\right|^{2}\right)^{1 / 2}\right\|_{C_{0}\left(K_{1}\right)}\left\|\left(\sum_{j=1}^{M}\left|g_{j}\right|^{2}\right)^{1 / 2}\right\|_{C_{j}\left(K_{2}\right)} .
$$

Similar arguments, now with $M=1$, yields

$$
\left\|\sum_{k=1}^{N} T\left(f_{k}, g_{k}\right)\right\|_{Y} \leq C\left\|\left(\sum_{k=1}^{N}\left|f_{k}\right|^{2}\right)^{1 / 2}\right\|_{C_{0}\left(K_{1}\right)}\left\|\left(\sum_{k=1}^{N}\left|g_{k}\right|^{2}\right)^{1 / 2}\right\|_{C_{0}\left(K_{2}\right)}
$$

as required.

Corollary 2.8. The following statements are equivalent for a Banach space valued factorable 1-summing bilinear operator $T: C_{0}\left(K_{1}\right) \times C_{0}\left(K_{2}\right) \rightarrow Y$ :

(i) For all finite sequences $\left(f_{j}\right)_{j=1}^{M}$ in $C_{0}\left(K_{1}\right)$ and $\left(g_{j}\right)_{j=1}^{M}$ in $C_{0}\left(K_{2}\right)$,

$$
\sum_{j=1}^{M}\left\|T\left(f_{j}, g_{j}\right)\right\|_{Y} \leq C\left\|\left(\sum_{j=1}^{M}\left|f_{j}\right|^{2}\right)^{1 / 2}\right\|_{C_{0}\left(K_{1}\right)}\left\|\left(\sum_{j=1}^{M}\left|g_{j}\right|^{2}\right)^{1 / 2}\right\|_{C_{0}\left(K_{2}\right)} .
$$

(ii) There are regular Borel measures $\lambda_{1}$ and $\lambda_{2}$ on $K_{1}$ and $K_{2}$, respectively, such that for every $f \in C_{0}\left(K_{1}\right)$ and $g \in C_{0}\left(K_{2}\right)$,

$$
\|T(f, g)\|_{Y} \leq C\left(\int_{K_{1}}|f|^{2} d \lambda_{1}\right)^{1 / 2}\left(\int_{K_{2}}|g|^{2} d \lambda_{2}\right)^{1 / 2} .
$$

In particular, this implies the following factorizations:


Proof. (i) is a direct consequence of Proposition 2.7(i) for $q=p=1$. The equivalence of (i) and (ii) can be proved using a standard Hahn-Banach separation argument (see for example [19]).

We remark here that not all Banach valued bilinear operators $C_{0}\left(K_{1}\right) \times C_{0}\left(K_{2}\right)$ are factorable 1-summing. To see this, note that there is a bounded bilinear functional $T: c_{0} \times c_{0} \rightarrow$ $\mathbb{K}$ such that

$$
\sum_{j, k=1}^{\infty}\left|T\left(e_{j}, e_{k}\right)\right|=\infty
$$

(see [18]). Clearly, such a $T$ is not factorable 1-summing; however it satisfies the Grothendieck Factorization Theorem. 
2.3. Summability of bilinear integrals operators. Many relevant multilinear operators appearing in classic and new problems of harmonic analysis and partial differential equations are essentially defined by means of the composition of a pointwise multiplication of functions and a kernel operator. This is the case for example of the so called bilinear Fourier integral operators or the bilinear oscillatory integrals (see, e.g., [4, 13]). For example, consider a classical bilinear Fourier integral operator $P_{\sigma}$ where $\sigma$ is a Hörmander symbol by

$$
P_{\sigma}\left(f_{1}, f_{2}\right)(x)=\int_{\mathbb{R}^{2 n}} \sigma\left(x, \xi_{1}, \xi_{2}\right) \widehat{f}_{1}\left(\xi_{1}\right) \widehat{f}_{2}\left(\xi_{2}\right) e^{2 \pi i x\left(\xi_{1}+\xi_{2}\right)} d \xi_{1} d \xi_{2}
$$

originally defined for $f_{1}, f_{2}$ Schwartz functions on $\mathbb{R}^{2 n}$. Here, as usual $\widehat{f}$ denotes the Fourier transform of the Schwartz function on $\mathbb{R}^{2 n}$ given by $\widehat{f}(\xi)=\int_{\mathbb{R}^{2 n}} f(x) e^{-2 \pi i(x, \xi)} d x$ for all $\xi \in \mathbb{R}^{2 n}$, where $(\cdot, \cdot)$ denotes the inner product in $\mathbb{R}^{2 n}$.

It can be easily seen that an equivalent formula for $P_{\sigma}$ is given by

$$
P_{\sigma}\left(f_{1}, f_{2}\right)(x)=\int_{\mathbb{R}^{4 n}} \sigma\left(x, \xi_{1}, \xi_{2}\right) f_{1}\left(y_{1}\right) f_{2}\left(y_{2}\right) e^{2 \pi i\left(\left(x-y_{1}\right) \xi_{1}+\left(x-y_{2}\right) \xi_{2}\right)} d y_{1} d y_{2} d \xi_{1} d \xi_{2},
$$

in which the pointwise multiplication appears explicitly.

To obtain suitable domains and ranges for such operators $-L^{p}$-spaces, weak $L^{p}$-spaces, Lorentz and Marcinkiewicz spaces - is the subject of some fundamental developments in harmonic analysis, as the Calderón-Zygmund Theory (see [14]). The arguments used for obtaining these spaces are deeply related to the classical developments in harmonic analysis, and in general only few of the known results in general multilinear operators summability theory are applied.

We will show that general functional analytic arguments as the ones obtained in this paper allow also to shed some light on the summability properties of such maps.

Lemma 2.9. Let $E$ and $Y$ be Banach spaces and let $(\Omega, \mathcal{A}, \mu)$ be a $\sigma$-finite measure. If $Y$ has cotype $2 \leq q<\infty$, then the following are equivalent statements about a blinear operator $T: L^{\infty}(\mu) \times E \rightarrow Y$.

(i) $T$ is factorable $(q, 1)$-summing

(ii) There exists a constant $C>0$ such that every finite sequences $\left(f_{j}\right)_{j=1}^{n}$ and $\left(x_{j}\right)_{j=1}^{n}$ in $L^{\infty}(\mu)$ and $X$, respectively, we have

$$
\left\|\sum_{j=1}^{n} T\left(f_{j}, x_{j}\right)\right\|_{Y} \leq \text { Cess } \sup _{\omega}\left\|\sum_{j=1}^{n} f_{j}(\omega) x_{j}\right\|_{E} .
$$

Proof. We will use Theorem 2.4 (ii). Recall that $L^{\infty} \otimes_{\varepsilon} E \hookrightarrow L^{\infty}(\mu, E)$ isometrically. Clearly, the inequality appearing in (ii) means that the linear extension $\widetilde{T}$ of $T$-originally defined in the projective tensor product- can be extended to $L^{\infty} \otimes_{\varepsilon} E$, since

$$
\text { ess } \sup _{\omega}\left\|\sum_{j=1}^{n} f_{j}(\omega) x_{j}\right\|_{E}=\left\|\sum_{j=1}^{n} f_{j} x_{j}\right\|_{L^{\infty}(\mu, E)} .
$$

On the other hand, $\widetilde{T}$ is $(q, 1)$-summing, since $Y$ has cotype $q$. Thus, Theorem 2.4 (ii) gives the required equivalence.

We present an example from classical analysis of a bilinear map that is defined by the composition of pointwise product of functions (of different variable) and a measurable kernel. 
Given three $\sigma$-finite and complete measure spaces $\left(\Omega_{1}, \Sigma_{1}, \mu\right),\left(\Omega_{2}, \Sigma_{2}, \nu\right),\left(\Omega_{3}, \Sigma_{3}, \eta\right)$ and a measurable kernel defined on $\Omega_{1} \times \Omega_{2} \times \Omega_{3}$, we define a bilinear map $I$ by

$$
I(f, g)(x):=\int_{\Omega_{1} \times \Omega_{2}} k(\omega, v, x) f(\omega) g(v) d \mu(\omega) d \nu(v), \quad x \in \Omega_{3},
$$

where $f$ and $g$ are measurable functions in the corresponding measure spaces.

Theorem 2.10. A bilinear integral I generated by a kernel $k$ is factorable $(q, 1)$-summing from the product $I: L^{\infty}(\mu) \times L^{r}(\nu) \rightarrow L^{q}(\eta)$ if and only if there exists a constant $C>0$ such that the kernel operator $K$ defined by

$$
K(t)(x):=\int_{\Omega_{1} \times \Omega_{2}} k(\omega, v, x) t(\omega, v) d \mu(\omega) d \nu(v), \quad x \in \Omega_{3}
$$

for all $t \in L^{\infty}(\mu) \otimes L^{r}(\nu)$ satisfies the condition

$$
\|K(t)\|_{L^{q}(\eta)} \leq C\|\| t(\omega, v)\left\|_{L^{r}(\nu)}\right\|_{L^{\infty}(\mu)} .
$$

Proof. For any finite sequence $\left(f_{i}\right)_{i=1}^{n}$ in $L^{\infty}(\mu)$ and $\left(g_{i}\right)_{i=1}^{n}$ in $L^{r}(\nu)$, we have

$$
\begin{aligned}
\left\|\sum_{j=1}^{n} I\left(f_{j}, g_{j}\right)\right\|_{L_{q}(\eta)} & =\left(\int_{\Omega_{3}}\left|\sum_{j=1}^{n} \int_{\Omega_{1} \times \Omega_{2}} k(\omega, v, x) f_{j}(\omega) g_{j}(v) d \mu(\omega) d \nu(v)\right|^{q} d \eta(x)\right)^{1 / q} \\
& =\left(\int_{\Omega_{3}}\left|\int_{\Omega_{1} \times \Omega_{2}} k(\omega, v, x)\left(\sum_{j=1}^{n} f_{j}(\omega) g_{j}(v)\right) d \mu(\omega) d \nu(v)\right|^{q} d \eta(x)\right)^{1 / q}
\end{aligned}
$$

and

$$
\text { ess } \sup _{\omega}\left\|\sum_{j=1}^{n} f_{j}(\omega) g_{j}\right\|_{L^{r}(\nu)}=e s s \sup _{\omega}\left(\int_{\Omega_{3}}\left|\sum_{j=1}^{n} f_{j}(\omega) g_{j}(v)\right|^{r} d \nu(v)\right)^{1 / r} .
$$

Then the inequality shown in the condition (ii) in Lemma 2.9 can be rewritten as the integral inequality

$$
\begin{gathered}
\left(\int_{\Omega_{3}}\left|\int_{\Omega_{1} \times \Omega_{2}} k(\omega, v, x)\left(\sum_{j=1}^{n} f_{j}(\omega) g_{j}(v)\right) d \mu(\omega) d \nu(v)\right|^{q} d \eta(x)\right)^{1 / q} \\
\leq \text { Cess } \sup _{\omega}\left(\int_{\Omega_{3}}\left|\sum_{j=1}^{n} f_{j}(\omega) g_{i}(v)\right|^{r} d \nu(v)\right)^{1 / r} .
\end{gathered}
$$

Combining with Lemma 2.9 we obtain the required statement.

2.4. Bilinear operators on the disk algebra. In this subsection, we will characterize the classes of bilinear operators as $T: X_{1} \times X_{2} \rightarrow Y$ that satisfy that the one-side associated linear operator $\Phi_{T}: X_{1} \rightarrow L\left(X_{2}, Y\right)$ has certain summability properties. We will show a characterization of the class of bilinear maps for which $\Phi_{T}$ is defined on the space $\Pi_{q, r}\left(X_{2}, Y\right)$ and is $(s, p)$-summing. As an application we will show variants of Mityagin-Pełczyński and Kislyakov Theorems on the existence of extensions to $C(\mathbb{T}) \times E$ preserving summability properties of bilinear operators acting in the product of the disc algebra $C_{A}$ and a Banach space E.

The class of bilinear operators given in Definition 2.11 below will be the key of our results of the section. It was initially inspired in [22] and also in how the class of bilinear operators introduced in Definition 3.6 in [33] is used for proving Proposition 3.7 in the same paper. Our definition is not symmetric, in the sense that we develop the "right hand side" version of 
such results; the reader will notice that a similar development can be done in the symmetric "left hand side" case.

Definition 2.11. Let $X_{1}, X_{2}, Y$ be Banach spaces, and let $1 \leq q, s, p, r \leq \infty$ such that $r \leq q$ and $p \leq s$. A bilinear operator $T: X_{1} \times X_{2} \rightarrow Y$ is said to be right- $(q, s ; p, r)$-summing if there is a constant $C>0$ such that for all sequences $\left(x_{j}^{(1)}\right)_{j=1}^{M}$ in $X_{1}$ and all $M \times N$ matrices $\left(x_{j k}^{(2)}\right)$ in $X_{2}$, we have

$$
\left(\sum_{j=1}^{M}\left(\sum_{k=1}^{N}\left\|T\left(x_{j}^{(1)}, x_{j k}^{(2)}\right)\right\|_{Y}^{q}\right)^{s / q}\right)^{1 / s} \leq C\left\|\left(x_{j}^{(1)}\right)\right\|_{w, p} \max _{1 \leq j \leq M}\left\|\left(x_{j k}^{(2)}\right)\right\|_{w, r} .
$$

Recall that given a bilinear operator $T: X_{1} \times X_{2} \rightarrow Y$, it defines a linear operator $\Phi_{T}: X_{1} \rightarrow L\left(X_{2}, Y\right)$ by the formula

$$
\Phi_{T}\left(x^{(1)}\right)=T\left(x^{(1)}, \cdot\right): X_{2} \rightarrow Y .
$$

Proposition 2.12. Let $T: X_{1} \times X_{2} \rightarrow Y$ be a bilinear operator. Then it is right- $(q, s ; p, r)$ summing if and only if the associated linear operator $\Phi_{T}$ is defined from $X_{1}$ to $\Pi_{q, r}\left(X_{2}, Y\right)$ and it is $(s, p)$-summing. In this case, the least constant $C$ in the definition coincides with $\pi_{s, p}\left(\Phi_{T}\right)$.

Proof. Suppose first that $T$ is right- $(q, s ; p, r)$-summing, and consider the linear map $\Phi_{T}$. Assume that $1 \leq q, s, p, r<\infty$ (the proof for the cases involving parameters equal to $\infty$ is the same and is obtained with the obvious changes). Fix $M=1$, and consider $x^{(1)} \in X_{1}$ and a sequence of elements $\left(x_{1 k}^{(2)}\right)_{k=1}^{N}$ in $X_{2}$. Then

$$
\left(\sum_{k=1}^{N}\left\|T\left(x^{(1)}, x_{1 k}^{(2)}\right)\right\|_{Y}^{q}\right)^{1 / q} \leq C\left\|x^{(1)}\right\|_{X_{1}}\left\|\left(x_{1 k}^{(2)}\right)\right\|_{w, r} .
$$

That is, the operator $\Phi_{T}\left(x^{(1)}\right): X_{2} \rightarrow Y$ is $(q, r)$-summing. On the other hand, for every $\varepsilon>0$ and every finite sequence $\left(x_{j}^{(1)}\right)_{j=1}^{M}$ in $X_{1}$ there exists a sequence $\left(x_{j k}^{(2)}\right)_{k=1}^{N}$ for each $1 \leq j \leq M$ such that $\left\|\left(x_{j k}^{(2)}\right)\right\|_{w, r} \leq 1$ and

$$
\begin{aligned}
\left(\sum_{j=1}^{M} \pi_{q, r}\left(\Phi_{T}\left(x_{j}^{(1)}\right)\right)^{s}\right)^{1 / s} & \leq(1+\varepsilon)\left(\sum_{j=1}^{M}\left(\sum_{k=1}^{N}\left\|T\left(x_{j}^{(1)}, x_{j k}^{(2)}\right)\right\|^{q}\right)^{s / q}\right)^{1 / s} \\
& \leq C(1+\varepsilon)\left\|\left(x_{j}^{(1)}\right)\right\|_{w, p} \max _{1 \leq j \leq M}\left\|\left(x_{j k}^{(2)}\right)\right\|_{w, r} \leq C(1+\varepsilon)\left\|\left(x_{j}^{(1)}\right)\right\|_{w, p} .
\end{aligned}
$$

Since $\varepsilon$ is arbitrary, $\Phi_{T}$ is $(s, p)$-summing with $\pi_{s, p}\left(\Phi_{T}\right) \leq C$.

For the converse, just take a sequence $\left(x_{j}^{(1)}\right)$ in $X_{1}$ and a matrix $\left(x_{j k}^{(2)}\right)$ in $X_{2}$. Then

$$
\begin{aligned}
\left(\sum_{j=1}^{M}\left(\sum_{k=1}^{N}\left\|T\left(x_{j}^{(1)}, x_{j k}^{(2)}\right)\right\|_{Y}^{q}\right)^{s / q}\right)^{1 / s} & =\left(\sum_{j=1}^{M}\left(\sum_{k=1}^{N}\left\|\Phi_{T}\left(x_{j}^{(1)}\right)\left(x_{j k}^{(2)}\right)\right\|_{Y}^{q}\right)^{s / q}\right)^{1 / s} \\
& \leq\left(\sum_{j=1}^{M} \pi_{q, r}\left(\left(\Phi_{T}\left(x_{j}^{(1)}\right)\right)^{s}\left\|\left(x_{j k}^{(2)}\right)\right\|_{w, r}^{s}\right)^{1 / s}\right. \\
& \leq \pi_{q, p}\left(\Phi_{T}\right)\left\|\left(x_{j}^{(1)}\right)\right\|_{w, p} \max _{1 \leq j \leq M}\left\|\left(x_{j k}^{(2)}\right)\right\|_{w, r} .
\end{aligned}
$$

The computations also show the coincidence of $C$ and $\pi_{s, p}\left(\Phi_{T}\right)$. 
We remark here that bilinear operators satisfying that the associated linear ones are defined in a space of $p$-summing operators and are $q$-summing has been already considered. In fact, they play a relevant role in [22], in which this property appears (see for instance Theorems 11 and 12 in this paper). Some of the bilinear maps considered act also in the product $C(K) \times E$, in which we will center our attention in what follows.

We also note that there is a connection between factorable $(q, p)$-summing operators and right- $(q, s ; p, r)$-summing operators. In particular, it can be easily seen by using Theorem 2.4 that Theorem 5 in [22] gives the following: if $X_{1}$ is an $L^{\infty}$-space, then for Banach spaces $X_{2}$ and $Y$, a bilinear operator $T: X_{1} \times X_{2} \rightarrow Y$ is factorable (1,1)-summing if and only if $\Phi_{T}: X_{1} \rightarrow \Pi_{1}\left(X_{2}, Y\right)$ is 1-summing. It follows from Proposition 2.12 that this can be rewritten as follows: a bilinear operator $T: L^{\infty} \times X_{2} \rightarrow Y$ is factorable (1,1)-summing if and only if it is right- $(1,1 ; 1,1)$-summing.

Theorem 6 in [22] gives also a coincidence result. In this case, it can be rewritten as follows: Let $X_{1}, X_{2}$ and $Y$ be Banach spaces. If $T: X_{1} \times X_{2} \rightarrow Y$ is a factorable (2,2)-summing operator, then $\Phi_{T}: X_{1} \rightarrow \Pi_{2}\left(X_{2}, Y\right)$ is a 2 -summing operator, that is, $T$ is right- $(2,2 ; 2,2)$ summing.

Taking into account that cotype $q(q \geq 2)$ for a Banach space $X$ implies that the identity map in $X$ is $(q, 1)$-summing, we obtain the following

Corollary 2.13. Suppose that the Banach space $Y$ has cotype q. Then all the classes of right- $(t, s ; p, r)$-summing bilinear operators for $t \geq q>r$ coincide with the one of right$(q, s ; p, 1)$-summing bilinear maps.

We apply our result to prove some variants of Mityagin-Pełczyński and Kislyakov Theorems on the existence of a bilinear extension of a bilinear map from $C_{A} \times E$ to $C(\mathbb{T}) \times E$ preserving its summability properties. Here as usual we denote by $C_{A}$ the disk algebra, which is the uniform closure of the analytic polynomials in the Banach space $C(\mathbb{T})$ of continuous functions on the unit circle $\mathbb{T}$ in the complex plane.

We recall that Mityagin-Pełczyński Theorem states (see [26]): For $1<p<\infty$, every $p$ summing operator from the disk algebra $C_{A}$ to a Banach space $Y$ extends to a p-summing operator from $C(\mathbb{T})$ to $Y$.

We also will need Kislyakov's Theorem (see [16]) which states: For an arbitrary Banach space $Y$ and $1 \leq p<q<\infty$, every $(q, p)$-summing operator from the disk algebra $C_{A}$ extends to a $(q, p)$-summing operator from $C(\mathbb{T})$ to $Y$.

The above results allows us to conclude that the class of right- $(q, s ; p, r)$-summing bilinear operators admit extensions from $C_{A}$ to $C(\mathbb{T})$.

Corollary 2.14. Let $E$ a Banach space. Let $T: C_{A} \times X_{2} \rightarrow Y$ be a right- $(q, s ; p, r)$ summing bilinear operator. Then it can be extended to a right- $(q, s ; p, r)$-summing bilinear map $\widetilde{T}: C(\mathbb{T}) \times E \rightarrow Y$.

Proof. As a consequence of Proposition 2.12, we have that $\Phi_{T}$ can be defined as an operator $\Phi_{T}: C_{A} \rightarrow \Pi_{q, r}(E, Y)$ and $\Phi_{T}$ is $(s, p)$-summing. The application of Mityagin-Pełczyński Theorem (for $s=p$ ) or of Kislyakov's Theorem (for $p<s$ ) provides an extension to $C(\mathbb{T}$ ) that is still $(s, p)$-summing. Using again Proposition 2.12, we obtain the result.

The case of right $(\infty, s ; p, \infty)$-summing bilinear operators - that is, $q=r=\infty$ - is relevant for our purposes. Clearly that a bilinear operator $T: X_{1} \times X_{2} \rightarrow Y$ is $(\infty, s ; p, \infty)$-summing whenever

$$
\left(\sum_{j=1}^{M}\left\|T\left(x_{j}^{(1)}, x_{j}^{(2)}\right)\right\|_{Y}^{s}\right)^{1 / s} \leq C\left\|\left(x_{j}^{(1)}\right)\right\|_{w, p} \max _{1 \leq j \leq M}\left\|x_{j}^{(2)}\right\|_{X_{2}}
$$


holds for all finite sequences $\left(x_{j}^{(1)}\right)_{j=1}^{M}$ and $\left(x_{j}^{(2)}\right)_{j=1}^{M}$ in $X_{1}$ and $X_{2}$, respectively.

Lemma 2.15. If $T$ is factorable $(s, p)$-summing, then it is right $(\infty, s ; p, \infty)$-summing, and so $(\infty, s ; p, r)$-summing for all $1 \leq r \leq \infty$.

Proof. If the definition of being factorable $(s, p)$-summing is taken only for $M \times 1$ matrices, we conclude that for all sequences $\left(x_{j}^{(1)}\right)$ and $\left(x_{j}^{(2)}\right)$ in $X_{1}$ and $X_{2}$, respectively, we have

$$
\begin{aligned}
\left(\sum_{j=1}^{M}\left\|T\left(x_{j}^{(1)}, x_{j}^{(2)}\right)\right\|_{Y}^{s}\right)^{1 / s} & \leq C \sup _{x_{1}^{*} \in B_{X_{1}^{*}}, x_{2}^{*} \in B_{X_{2}^{*}}}\left(\sum_{j=1}^{M}\left|\left\langle x_{j}^{(1)}, x_{1}^{*}\right\rangle\left\langle x_{j}^{(2)}, x_{2}^{*}\right\rangle\right|^{p}\right)^{1 / p} \\
& \leq C \sup _{x_{1}^{*} \in B_{X_{1}^{*}}}\left(\sum_{j=1}^{M}\left|\left\langle x_{j}^{(1)}, x_{1}^{*}\right\rangle\right|^{p}\left\|x_{j}^{(2)}\right\|^{p}\right)^{1 / p} \\
& \leq C\left\|\left(x_{j}^{(1)}\right)\right\|_{w, p} \max _{1 \leq j \leq M}\left\|x_{j}^{(2)}\right\|_{X_{2}}
\end{aligned}
$$

and so the result follows.

A direct consequence of Corollaries 2.13 and 2.14 and Lemma 2.15 is the following

Corollary 2.16. If $T: C_{A} \times E \rightarrow Y$ is factorable $(s, p)$-summing, then there is an extension $\widetilde{T}: C(\mathbb{T}) \times E \rightarrow Y$ that is $(\infty, s ; p, r)$-summing for all $1 \leq r \leq \infty$. Moreover, if $Y$ is a cotype $q$ space, then $T$ is right $(q, s ; p, r)$-summing for all $1 \leq r<q$.

\section{FACTORIZATiOn of $(r ; p, q)$-SUMming BiLINEAR OPERATORS}

In this section we are interested in studying the factorization properties of $(r ; p, q)$-summing bilinear operators, where $r, p, q \in[1, \infty)$ and $1 / r \leq 1 / p+1 / q$. We will show that in this case domination and factorization do not follow the same lines.

We recall that the notion of $\left(q ; p_{1}, \ldots, p_{n}\right)$-summing operator was introduced in [2]. For more information we refer to the survey paper on multilinear summing operators [25]. The case $1 / q=1 / p_{1}+\cdots+1 / p_{n}$ has a particular interest in the theory of multilinear operators, and provides the so called "dominated operators". This notion defines a class of multilinear operators for which a Pietsch's Domination Theorem holds. It should be noticed here that this type of domination theorem for an $n$-linear operator $T: X_{1} \times \cdots \times X_{n} \rightarrow Y$ is equivalent to a factorization that is essentially different from the one that we have proved in the previous section. In the case of the mentioned Pietsch Domination Theorem, the operator factors through a product $L^{p_{1}}\left(\mu_{1}\right) \times \cdots \times L^{p_{n}}\left(\mu_{n}\right)$, where the $\mu_{i}$ 's are probability Borel measures on $B_{X_{i}^{*}}$ endowed with the weak* topology, by means of a product of canonical maps $j_{i}: C\left(B_{X_{i}^{*}}\right) \rightarrow L^{p_{i}}\left(\mu_{i}\right)$ for each $1 \leq i \leq n$. Thus the factorization is obtained by using a domination theorem involving probability measures and functions in the unit balls of the corresponding $C(K)$-spaces, as in the linear case (see [10, Theorem 10.8]). We will show in this section that these ideas do not work for the general case of summing multilinear maps. The interested reader can find different versions of domination/factorization theorems for dominated-type multilinear maps that extend the linear case in $[6,25,27]$ and the references therein.

We will need the following result from [19] on domination of bilinear operators.

Theorem 3.1. Let $0<p, q<\infty$ and let $X$ and $Y$ be quasi-Banach lattices such that both duals $\left(X_{1 / p}\right)^{*}$ and $\left(Y_{1 / q}\right)^{*}$ separates the points of $X_{1 / p}$ and $Y_{1 / q}$, respectively. Assume $\phi \in \Phi$, $0<C_{1}, C_{2}<\infty$ and that $A \subset X, B \subset Y$ are non-empty sets. The following are equivalent statements about a bilinear operator $T$ from $X \times Y$ to a quasi-Banach space $E$. 
(i) For any set of positive scalars $\left(\alpha_{k}\right)_{k=1}^{n}$ with $\sum_{k=1}^{n} \alpha_{k}=1$ and any sets $\left(f_{k}\right)_{k=1}^{n}$ in $A$ and $\left(g_{k}\right)_{k=1}^{n}$ in $B, n \in \mathbb{N}$,

$$
\sum_{k=1}^{n} \alpha_{k} \phi\left(\left\|T\left(f_{k}, g_{k}\right)\right\|_{E}\right) \leq C_{1}\left\|\sum_{k=1}^{n} \alpha_{k}\left|f_{k}\right|^{p}\right\|_{X_{1 / p}}^{c}+C_{2}\left\|\sum_{k=1}^{n} \alpha_{k}\left|g_{k}\right|^{q}\right\|_{Y_{1 / q}}^{c} .
$$

(ii) There exist positive functionals $x^{*} \in B_{\left(X_{1 / p}\right)^{*}}$ and $y^{*} \in B_{\left(Y_{1 / q}\right)^{*}}$ such that

$$
\phi\left(\|T(f, g)\|_{E}\right) \leq C_{1} x^{*}\left(|f|^{p}\right)+C_{2} y^{*}\left(|g|^{q}\right), \quad(f, g) \in A \times B .
$$

Here as usual given a quasi-Banach lattice $X$ and $0<s<\infty, X_{s}$ is the $s$-convexification $X_{s}$ of $X$ equipped with the quasi-norm $\|x\|_{X_{s}}=\left\||x|^{s}\right\|_{X}^{1 / s}$, and $\|\cdot\|_{X_{1 / p}}^{c}$ denotes the Mackey norm on $X_{1 / p}$.

Let $1 \leq r, p, q<\infty$ be such that $1 / r \leq 1 / p+1 / q$. We start our discussion on $(r ; p, q)$ summing bilinear operators from the product of $C(K)$-spaces. Since for every $1 \leq s<\infty$ and any choice of finite sequences $\left(f_{k}\right)_{k=1}^{n}$ in $C(K)$,

$$
\sup _{\|\varphi\|_{C(K)^{*} \leq 1}}\left(\sum_{k=1}^{n}\left|\left\langle f_{k}, \varphi\right\rangle\right|^{s}\right)^{1 / s}=\left\|\left(\sum_{k=1}^{n}\left|f_{k}\right|^{s}\right)^{1 / s}\right\|_{C(K)},
$$

it follows that a Banach space valued bilinear operator $T: C\left(K_{1}\right) \times C\left(K_{2}\right) \rightarrow Y$ is $(r ; p, q)$ summing if there is a constant $C>0$ such that for any choice of finite sequences elements $\left(f_{k}\right)_{k=1}^{n}$ in $C\left(K_{1}\right)$ and $\left(g_{k}\right)_{k=1}^{n}$ in $C\left(K_{2}\right)$,

$$
\left(\sum_{k=1}^{n}\left\|T\left(f_{k}, g_{k}\right)\right\|^{r}\right)^{1 / r} \leq C\left\|\left(\sum_{k=1}^{n}\left|f_{k}\right|^{p}\right)^{1 / p}\right\|_{C\left(K_{1}\right)}\left\|\left(\sum_{k=1}^{n}\left|g_{k}\right|^{q}\right)^{1 / q}\right\|_{C\left(K_{2}\right)}
$$

We observe that it follows from factorization Theorem 2.5 that an operator $T$ satisfying the summability property shown in condition (i) is also $(r ; p, q)$-summing, $1 / r<1 / p+1 / q, r \geq 1$. To see this, use Pisier's Theorem for the inclusion $C\left(K_{1}\right) \otimes_{\epsilon} C\left(K_{2}\right)=C\left(K_{1} \times K_{2}\right) \rightarrow L_{q, 1}(\mu)$ : since it is $(r, 1)$-summing (see [10, p. 205]), we conclude that for any pair of finite sequences $\left(f_{j}\right)_{j=1}^{n}$ in $C\left(K_{1}\right)$ and $\left(g_{j}\right)_{j=1}^{n}$ in $C\left(K_{2}\right)$, we have

$$
\begin{gathered}
\left(\sum_{k=1}^{n}\left\|\odot\left(f_{k}, g_{k}\right)\right\|_{L_{r, 1}(\mu)}^{r}\right)^{1 / r} \leq C\left\|\sum_{k=1}^{n}\left|\odot\left(f_{k}, g_{k}\right)\right|\right\|_{C\left(K_{1} \times K_{2}\right)} \\
\leq C\left\|\left(\sum_{k=1}^{n}\left|f_{k}\right|^{p}\right)^{1 / p}\left(\sum_{k=1}^{n}\left|g_{k}\right|^{q}\right)^{1 / q}\right\| \|_{C\left(K_{1} \times K_{2}\right)} \\
\leq C\left\|\left(\sum_{k=1}^{n}\left|f_{k}\right|^{p}\right)^{1 / p}\right\|_{C\left(K_{1}\right)}\left\|\left(\sum_{k=1}^{n}\left|g_{k}\right|^{q}\right)^{1 / q}\right\|_{C\left(K_{2}\right)}
\end{gathered}
$$

and so combining with factorization we obtain that $T$ is $(r ; p, q)$-summing.

Let us show now our first example. Let $T: X \times Y \rightarrow Z$ be a bilinear operator from a product of Banach spaces in a Banach space $Z$, and let $1 \leq r<\infty, 2 \leq p, q<\infty$ be such that $1 / r \leq 1 / p+1 / q$. Assume that $X$ has cotype $p$ and $Y$ has cotype $q$. We claim that $T$ is $(r, 1,1)$-summing; in fact for any $x_{1}, \ldots, x_{n}$ in $X$ and $y_{1}, \ldots, y_{n}$ in $Y$, we have with 
$C=\|T\| C_{p}(X) C_{q}(Y)$

$$
\begin{aligned}
\left(\sum_{k=1}^{n}\left\|T\left(x_{k}, y_{k}\right)\right\|_{Z}^{r}\right)^{1 / r} & \leq\|T\|\left(\sum_{k=1}^{n}\left\|x_{k}\right\|^{p}\right)^{1 / p}\left(\sum_{k=1}^{n}\left\|x_{k}\right\|^{q}\right)^{1 / q} \\
& \leq C\left(\int_{0}^{1}\left\|\sum_{k=1}^{n} r_{k}(t) x_{k}\right\|_{X}^{2} d t\right)^{1 / 2}\left(\int_{0}^{1}\left\|\sum_{k=1}^{n} r_{k}(t) y_{k}\right\|_{Y}^{2} d t\right)^{1 / 2} \\
& \leq C \sup _{\left|\epsilon_{k}\right|=1}\left\|\sum_{k=1}^{n} \epsilon_{k} x_{k}\right\|_{X} \sup _{\left|\varepsilon_{k}\right|=1}\left\|\sum_{k=1}^{n} \varepsilon_{k} y_{k}\right\|_{Y} \\
& =C \sup _{\left\|x^{*}\right\|_{X^{*}} \leq 1}\left(\sum_{k=1}^{n}\left|\left\langle x_{k}, x^{*}\right\rangle\right|\right) \sup _{\left\|y^{*}\right\|_{Y^{*}} \leq 1}\left(\sum_{k=1}^{n}\left|\left\langle y_{k}, y^{*}\right\rangle\right|\right) .
\end{aligned}
$$

To show the next example assume that $1 \leq r \leq p, q<\infty$ such that $1 / r=1 / p+1 / q$ and let $T: X \times Y \rightarrow Z$ be a positive bilinear operator from a product of Banach lattices into an $r$-concave Banach lattice $Z$ with constant $M^{(r)}(Z)=1$. Using the inequality given in [8, Theorem 6.2], we conclude that for any choice of elements $x_{1}, \ldots, x_{n}$ in $X$ and $y_{1}, \ldots, y_{n}$ in $Y$, we have (with $C=\|T\| C_{p}(X) C_{q}(Y)$ ),

$$
\begin{aligned}
\left(\sum_{k=1}^{n}\left\|T\left(x_{k}, y_{k}\right)\right\|_{Z}^{r}\right)^{1 / r} & \leq\|T\|\left(\sum_{k=1}^{n}\left\|x_{k}\right\|_{X}^{p}\right)^{1 / p}\left(\sum_{k=1}^{n}\left\|y_{k}\right\|_{Y}^{q}\right)^{1 / q} \\
& \leq C\left(\int_{0}^{1}\left\|\sum_{k=1}^{n} r_{k}(t) x_{k}\right\|_{X}^{2} d t\right)^{1 / 2}\left(\int_{0}^{1}\left\|\sum_{k=1}^{n} r_{k}(t) y_{k}\right\|_{Y}^{2} d t\right)^{1 / 2} \\
& \leq C \sup _{\left|\varepsilon_{k}\right|=1}\left\|\sum_{k=1}^{n} \varepsilon_{k} x_{k}\right\|_{X} \sup _{\left|\varepsilon_{k}\right|=1}\left\|\sum_{k=1}^{n} \varepsilon_{k} y_{k}\right\|_{Y} \\
& =C \sup _{\left\|x^{*}\right\|_{X^{*}} \leq 1}\left(\sum_{k=1}^{n}\left|\left\langle x_{k}, x^{*}\right\rangle\right|\right) \sup _{\left\|y^{*}\right\|_{Y^{*}} \leq 1}\left(\sum_{k=1}^{n}\left|\left\langle y_{k}, y^{*}\right\rangle\right|\right) .
\end{aligned}
$$

This shows that $T$ is $(r ; p, q)$-summing.

As it was mentioned for the general case at the beginning of this section, for $1 / r=$ $1 / p+1 / q$ the $(r ; p, q)$-summing bilinear operators satisfy a Pietsch's Domination/Factorization Theorem; more precisely a bilinear operator $T: C\left(K_{1}\right) \times C\left(K_{2}\right) \rightarrow Y$ is $(r ; p, q)$-summing if and only if it admits the following factorization:

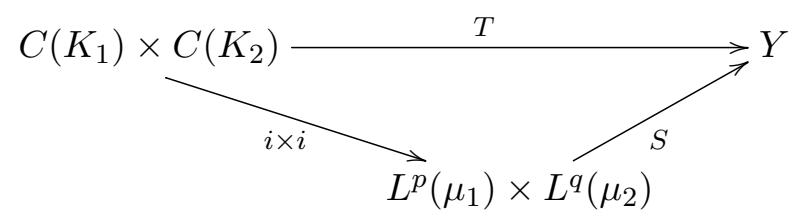

where $\mu_{1}$ and $\mu_{2}$ are probability Borel measures on $K_{1}$ and $K_{2}$, respectively (see $[12,21]$ ).

One could expect that the similar bilinear version is also true for Pisier's Theorem, i.e., if $1 / r<1 / p+1 / q$, then a bilinear operator $T$ from the product $C\left(K_{1}\right) \times C\left(K_{2}\right)$ to a Banach space $Y$ is $(r ; p, q)$-summing if and only if it factors as follows




for suitable probability Borel measures $\mu_{1}$ and $\mu_{2}$ on $K_{1}$ and $K_{2}$, respectively, and suitable $1 \leq s, t<\infty$. We show that this is not true in general, even if we assume that $T$ is a positive bilinear operator. Recall that the product $L_{s, 1}\left(\mu_{1}\right) \times L_{t, 1}\left(\mu_{2}\right)$ equipped with the natural order and norm is an order continuous Banach lattice, and the same is true with the product of any pair of order continuous Banach lattices.

Proposition 3.2. Let $1 \leq p<\infty$. There is a $(1 ; p, 1)$-summing positive bilinear map $T: C(K) \times C(K) \rightarrow C(K)$ which does not admit a factorization:

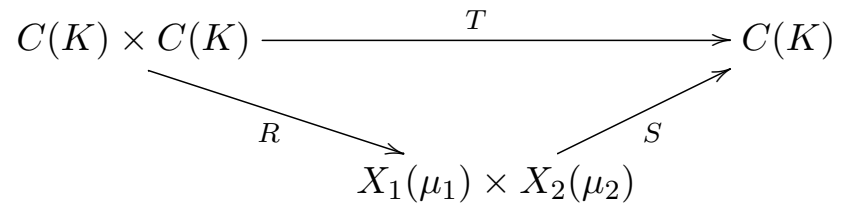

for any pair of order continuous Banach lattices $X_{1}\left(\mu_{1}\right) \subset L^{1}\left(\mu_{1}\right)$ and $X_{2}\left(\mu_{2}\right) \subset L^{1}\left(\mu_{2}\right)$ over finite measure spaces, any positive linear operator $R$ and any bilinear operator $S$. In particular, we conclude that there is a positive $(1 ; p, 1)$-summing bilinear operator which does not factor through $L_{s, 1}\left(\mu_{1}\right) \times L_{t, 1}\left(\mu_{2}\right)$ for any pair of probability Borel measures $\mu_{1}$ and $\mu_{2}$ on $K_{1}$ and $K_{2}$, respectively, and suitable $1 \leq s, t<\infty$.

Proof. Let $\varphi \in C(K)^{*}$ be a positive norm one functional on $C(K)$. Consider the bilinear operator $T: C(K) \times C(K) \rightarrow C(K)$ defined by

$$
T(f, g)=\langle g, \varphi\rangle f, \quad(f, g) \in C(K) \times C(K) .
$$

Note that for any choice of finitely many $f_{1}, \ldots, f_{n}$ and $g_{1}, \ldots, g_{n}$ in $C(K)$, we have

$$
\begin{aligned}
\sum_{k=1}^{n}\left\|T\left(f_{k}, g_{k}\right)\right\|_{C(K)} & \leq \sum_{k=1}^{n}\left\|f_{k}\right\|_{C(K)}\left|\varphi\left(g_{k}\right)\right| \leq \sup _{1 \leq k \leq n}\left\|f_{k}\right\|_{C(K)}\left(\sum_{k=1}^{n}\left|\varphi\left(g_{k}\right)\right|\right) \\
& \leq\left\|\left(\sum_{k=1}^{n}\left|f_{k}\right|^{p}\right)^{1 / p}\right\|_{C(K)} \sup _{\phi \in B_{C(K)^{*}}}\left(\sum_{k=1}^{n}\left|\phi\left(g_{k}\right)\right|\right) \\
& =\left\|\left(\sum_{k=1}^{n}\left|f_{k}\right|^{p}\right)^{1 / p}\right\|_{C(K)}\left\|\sum_{k=1}^{n}\left|g_{k}\right|\right\|_{C(K)}
\end{aligned}
$$

and so $T$ is $(1 ; p, 1)$-summing. Suppose that $T$ admits a factorization:

$$
T: C(K) \times C(K) \stackrel{R}{\longrightarrow} X_{1}\left(\mu_{1}\right) \times X_{2}\left(\mu_{2}\right) \stackrel{S}{\longrightarrow} C(K)
$$

where $X_{1}\left(\mu_{1}\right)$ and $X_{2}\left(\mu_{2}\right)$ are Banach lattices with the properties appearing in the statement of the theorem, and $R$ is a positive linear operator. We recall the well known fact that a Banach is lattice $E$ is order continuous if and only if every order interval of $E$ is weakly compact (see [3, Theorem 12.9, p. 179]). Since $B_{C(K)} \times B_{C(K)}$ is order bounded in the order continuous Banach lattice $X_{1}\left(\mu_{1}\right) \times X_{2}\left(\mu_{2}\right)$, the image by the positive map $R$ is contained in an interval $I$ in $X_{1}\left(\mu_{1}\right) \times X_{2}\left(\mu_{2}\right)$ and so this would imply that $T$ is a weakly compact operator. However this is a contradiction by $T\left(B_{C(K)} \times B_{C(K)}\right)=B_{C(K)}$. As a consequence, we conclude that $T$ does not admit a factorization through $L_{s, 1}\left(\mu_{1}\right) \times L_{t, 1}\left(\mu_{2}\right)$ for any pair of probability Borel measures $\mu_{1}$ and $\mu_{2}$ on $K_{1}$ and $K_{2}$, respectively, and $1 \leq t, s \leq \infty$.

We remark that the bilinear operator $C(K) \times C(K) \ni(f, g) \longmapsto\langle g, \varphi\rangle P(f)$, where $P$ is any positive non weakly compact operator from $C(K)$ to a Banach lattice $Y$, provides a new counterexample to the factorization problem discussed in the above proof. Motivated by 
this negative result we will consider the question of factorization of bilinear $(r ; p, q)$-summing bilinear operators from the product of $C(K)$-spaces.

We start with the following remark: if $1 \leq p<r<\infty$ and $1 \leq q<r$, then a bilinear operator $T$ from the product $C\left(K_{1}\right) \times C\left(K_{2}\right)$ to a Banach space which factors through the product $L_{r, 1}\left(\mu_{1}\right) \times L_{r, 1}\left(\mu_{2}\right)$ of Lorentz spaces is $(r ; p, q)$-summing. To see this we need to use Theorem 3.3, that will be proved later. If $f \in B_{C\left(K_{1}\right)}$ and $g \in B_{C\left(K_{2}\right)}$, it follows from the linear Pisier's Factorization Theorem that there exists a positive constant $C$ such that

$$
\|T(f, g)\|^{r} \leq\|S\|^{r}\left(\|f\|_{L_{r, 1}\left(\mu_{1}\right)}+\|g\|_{L_{r, 1}\left(\mu_{2}\right)}\right)^{r} \leq C\left(\|f\|_{L^{p}\left(\mu_{1}\right)}^{p}+\|g\|_{L^{q}\left(\mu_{2}\right)}^{q}\right) .
$$

Thus applying Theorem 3.3 we conclude that $T$ is $(r ; p, q)$-summing.

To prove and state our next results, we need to define some Banach spaces which seem to be of independent interest. Fix $p, q, r \in[1, \infty)$ with $1 \leq p<r, 1 \leq q<r$ and compact Hausdorff spaces $K_{1}$ and $K_{2}$, and two probability Borel measures $\mu_{1}$ and $\mu_{2}$ on $K_{1}$ and $K_{2}$, respectively. We define a seminorm $\pi_{r ; p, q}$ on the tensor product $C\left(K_{1}\right) \otimes C\left(K_{2}\right)$ by the formula:

$$
\pi_{r ; p, q}(z):=\inf \left\{\sum_{k=1}^{n}\left(\left\|f_{k}\right\|_{L^{p}\left(\mu_{1}\right)}^{p / r}\left\|f_{k}\right\|_{C\left(K_{1}\right)}^{1-p / r}\left\|g_{k}\right\|_{C\left(K_{2}\right)}+\left\|g_{k}\right\|_{L^{q}\left(\mu_{2}\right)}^{q / r}\left\|g_{k}\right\|_{C\left(K_{2}\right)}^{1-q / r}\left\|f_{k}\right\|_{C\left(K_{1}\right)}\right)\right\}
$$

where the infimum is taken over all representations $z=\sum_{k=1}^{n} f_{k} g_{k}$ of $z \in C\left(K_{1}\right) \otimes C\left(K_{2}\right)$. We define the tensor product $C\left(K_{1}\right) \otimes_{\pi_{r ; p, q}} C\left(K_{2}\right)$ as the quotient space generated by the seminorm, and $C\left(K_{1}\right) \widehat{\otimes}_{\pi_{r ; p, q}} C\left(K_{2}\right)$ for its completion.

Now, we define a Banach lattice which will be a cornerstone for establishing our result on factorization of some class of $(r ; p, q)$-summing bilinear operators. The idea is based on a variant of the Fremlin $|\pi|$-tensor product for Banach lattices. Recall the definition of the positive projective norm $|\phi|$ on a tensor product of Banach lattices given in [11] and compare the definition below with [11, $1 \mathrm{E}(\mathrm{vi})]$. Let $p, q, r \in[1, \infty)$ satisfy $1 \leq p<r, 1 \leq q<r$. Consider two given probability Borel measures $\mu_{1}$ and $\mu_{2}$ in $K_{1}$ and $K_{2}$, respectively. In the tensor product $C\left(K_{1}\right) \otimes C\left(K_{2}\right)$ considered as a subspace of the Fremlin tensor product $C\left(K_{1}\right) \widehat{\otimes}_{|\pi|} C\left(K_{2}\right)$, we define a seminorm $|\pi|_{r ; p, q}$ by

$$
|\pi|_{r ; p, q}(z):=\inf \left\{\sum_{k=1}^{n}\left(\left\|f_{k}\right\|_{L^{p}\left(\mu_{1}\right)}^{p / r}\left\|f_{k}\right\|_{C\left(K_{1}\right)}^{1-p / r}\left\|g_{k}\right\|_{C\left(K_{2}\right)}+\left\|g_{k}\right\|_{L^{q}\left(\mu_{2}\right)}^{q / r}\left\|g_{k}\right\|_{C\left(K_{2}\right)}^{1-q / r}\left\|f_{k}\right\|_{C\left(K_{1}\right)}\right)\right\},
$$

where the infimum is taken over all dominations $|z| \leq \sum_{k=1}^{n}\left|f_{k} g_{k}\right|$. Notice that this seminorm is continuous with respect to $|\pi|$. Thus, the kernel of the seminorm is closed in the normed lattice. With the order induced from the normed lattice $C\left(K_{1}\right) \otimes_{|\pi|} C\left(K_{2}\right)$, it is clearly an ideal. We put $L_{r ; p, q}\left(\mu_{1}, \mu_{2}\right)_{0}$ for the quotient Riesz space, that is a normed Riesz space with the quotient lattice norm (see [3, Ch.4]). Note that it is also a normed Riesz space for the seminorm $|\pi|_{r ; p, q}$ that by definition of the quotient is also an order preserving norm in this space. Moreover, its completion is a Banach lattice (see [3, Theorem 4.2]). Clearly, in the dense (quotient) subspace $C\left(K_{1}\right) \otimes_{|\pi|_{r ; p, q}} C\left(K_{2}\right)$ of $L_{r ; p, q}\left(\mu_{1}, \mu_{2}\right)$ functions are identified with their $|\pi|_{r ; p, q}$ equivalence classes. Consequently, the positive bilinear map $\otimes: C\left(K_{1}\right) \times C\left(K_{2}\right) \rightarrow$ $C\left(K_{1}\right) \otimes_{|\pi|_{r ; p, q}} C\left(K_{2}\right) \subset L_{r ; p, q}\left(\mu_{1}, \mu_{2}\right)$ is well-defined.

We are now ready to state and prove the main result of this section.

Theorem 3.3. Let $1 \leq p, q, r<\infty$ satisfy $1 \leq p<r$ and $1 \leq q<r$. The following are equivalent statements about a Banach space valued bilinear operator $T: C\left(K_{1}\right) \times C\left(K_{2}\right) \rightarrow Y$. 
(i) There are probability Borel measures $\mu_{1}$ and $\mu_{2}$ on $K_{1}$ and $K_{2}$ and a constant $C>0$ such that for every $f \in B_{C\left(K_{1}\right)}$ and $g \in B_{C\left(K_{2}\right)}$,

$$
\|T(f, g)\|^{r} \leq C\left(\int_{K_{1}}|f|^{p} d \mu_{1}+\int_{K_{2}}|g|^{q} d \mu_{2}\right) .
$$

(ii) For every finite sequence of positive scalars $\left(\alpha_{k}\right)_{k=1}^{n}$ with $\sum_{k=1}^{n} \alpha_{k}=1$ and for any finite sequences $\left(f_{k}\right)_{k=1}^{n}$ in $B_{C\left(K_{1}\right)}$ and $\left(g_{k}\right)_{k=1}^{n}$ in $B_{C\left(K_{2}\right)}$, the following inequality holds

$$
\sum_{k=1}^{n} \alpha_{k}\left\|T\left(f_{k}, g_{k}\right)\right\|^{r} \leq C_{1}\left\|\sum_{k=1}^{n} \alpha_{k}\left|f_{k}\right|^{p}\right\|_{C\left(K_{1}\right)}+C_{2}\left\|\sum_{k=1}^{n} \alpha_{k}\left|g_{k}\right|^{q}\right\|_{C\left(K_{2}\right)} .
$$

(iii) $T$ admits the following factorization:

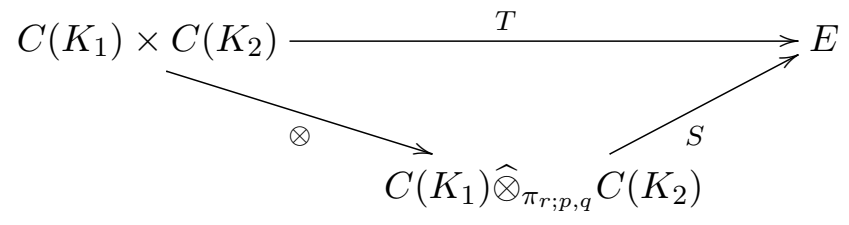

where $S$ a bounded linear operator.

Moreover, each of the above conditions implies

(iv) $T$ is $(r ; p, q)$-summing operator.

All conditions are equivalent whenever $T$ is a positive operator.

Proof. The equivalence of (i) and (ii) is given by Theorem 3.1 and the fact that $C(K)_{1 / s}=$ $C(K)$ for all $0<s<\infty$. For (ii) $\Rightarrow$ (iv), take two sequences of functions $\left(f_{k}\right)_{k=1}^{n}$ in $C\left(K_{1}\right)$ and $\left(g_{k}\right)_{k=1}^{n}$ in $C\left(K_{2}\right)$ such that $\sum_{k=1}^{n}\left|f_{k}\right|^{p} \leq 1$ and $\sum_{k=1}^{n}\left|g_{k}\right|^{p} \leq 1$. Then

$$
\begin{array}{r}
\sum_{k=1}^{n}\left\|T\left(f_{k}, g_{k}\right)\right\|^{r} \leq C\left(\int_{K_{1}} \sum_{k=1}^{n}\left|f_{k}\right|^{p} d \mu_{1}+\int_{K_{2}} \sum_{k=1}^{n}\left|g_{k}\right|^{q} d \mu_{2}\right) \\
\leq C\left(\left\|\sum_{k=1}^{n}\left|f_{k}\right|^{p}\right\|_{C\left(K_{1}\right)}+\left\|\sum_{k=1}^{n}\left|g_{k}\right|^{q}\right\|_{C\left(K_{2}\right)}\right) \leq 2 C .
\end{array}
$$

This clearly gives (iv).

(ii) $\Rightarrow$ (iii). By homogeneity of the norms involved and using the inequality $(a+b)^{t} \leq a^{t}+b^{t}$ for $0<t \leq 1$ and $a, b \geq 0$, we conclude by (ii) that for every pair $(f, g) \in C\left(K_{1}\right) \times C\left(K_{2}\right)$,

$$
\|T(f, g)\| \leq C\left(\|f\|_{L^{p}\left(K_{1}, \mu_{1}\right)}^{p / r}\|f\|_{C\left(K_{1}\right)}^{1-p / r}\|g\|_{C\left(K_{2}\right)}+\|g\|_{L^{q}\left(K_{2}, \mu_{2}\right)}^{q / r}\|g\|_{C\left(K_{2}\right)}^{1-q / r}\|f\|_{C\left(K_{1}\right)}\right) .
$$

Let $S: C\left(K_{1}\right) \otimes C\left(K_{2}\right) \rightarrow E$ be an operator given by $S(f \otimes g):=T(f, g)$ for any $f \otimes g \in$ $C\left(K_{1}\right) \otimes C\left(K_{2}\right)$. Then the above inequality implies that for every simple tensor $z=\sum_{k=1}^{n} f_{k} \otimes$ $g_{k} \in C\left(K_{1}\right) \otimes C\left(K_{2}\right)$ we have the inequalities

$$
\begin{aligned}
& \|S z\|_{Y} \leq\left\|\sum_{k=1}^{n} S\left(f_{k} \otimes g_{k}\right)\right\|_{Y}=\left\|\sum_{k=1}^{n} T\left(f_{k}, g_{k}\right)\right\|_{Y} \leq \sum_{k=1}^{n}\left\|T\left(f_{k}, g_{k}\right)\right\|_{Y} \\
& \leq C \sum_{k=1}^{n}\left(\left\|f_{k}\right\|_{L^{p}\left(K_{1}, \mu_{1}\right)}^{p / r}\left\|f_{k}\right\|_{C\left(K_{1}\right)}^{1-p / r}\left\|g_{k}\right\|_{C\left(K_{2}\right)}+\left\|g_{k}\right\|_{L^{q}\left(K_{2}, \mu_{2}\right)}^{q / r}\left\|g_{k}\right\|_{C\left(K_{2}\right)}^{1-q / r}\left\|f_{k}\right\|_{C\left(K_{1}\right)}\right) .
\end{aligned}
$$

This shows that $\|S z\|_{Y} \leq C \pi_{r ; p, q}(z)$ and so yields the required factorization. The converse statement is obvious. 
(iv) $\Rightarrow$ (i). Since $T$ is positive, we may assume without loss of generality that the $(r ; p, q)$ summing constant $C$ of $T$ equals 1 . Let $n \in \mathbb{N}$ and call $C_{n}$ to the $n$-vectors $(r ; p, q)$-summing constant of $T$. Notice that $C_{n} \leq 1$ and $\lim _{n} C_{n}=1$. Let $f_{1}, \ldots, f_{n} \in C\left(K_{1}\right)$ and $g_{1}, \ldots, g_{n} \in$ $C\left(K_{2}\right)$ be such that

$$
\left\|\left(\sum_{k=1}^{n}\left|f_{k}\right|^{p}\right)^{1 / p}\right\|_{C\left(K_{1}\right)} C_{n}(1-1 / n) \leq 1, \quad\left\|\left(\sum_{k=1}^{n}\left|g_{k}\right|^{q}\right)^{1 / q}\right\|_{C\left(K_{2}\right)} C_{n}(1-1 / n) \leq 1,
$$

and

$$
\sum_{k=1}^{n}\left\|T\left(f_{k}, g_{k}\right)\right\|^{r}=1
$$

Then there are elements $b_{k}^{*} \in Y^{*}$ such that for $1 / r+1 / r^{\prime}=1$, we have $\sum_{k=1}^{n}\left\|b_{k}^{*}\right\|^{r^{\prime}}=1$,

$$
\left(\sum_{k=1}^{n}\left\langle T\left(f_{k}, g_{k}\right), b_{k}^{*}\right\rangle\right)^{r}=\sum_{k=1}^{n}\left\|T\left(f_{k}, g_{k}\right)\right\|^{r}=1 .
$$

Since the bilinear map is positive, we can assume also that all the elements $\left(f_{k}, g_{k}\right.$ and $\left.b_{k}^{*}\right)$ in this expression are positive.

We use some ideas from the proof of Pisier's Factorization Theorem for linear operators (see [31]); we define a positive bilinear form $\nu: C\left(K_{1}\right) \times C\left(K_{2}\right) \rightarrow \mathbb{R}$ by the formula

$$
\nu(f, g):=\sum_{k=1}^{n}\left\langle T\left(f_{k} f, g_{k} g\right), b_{k}^{*}\right\rangle, \quad f \in C\left(K_{1}\right), g \in C\left(K_{2}\right) .
$$

Clearly, $\nu$ is bounded with norm less or equal than one.

Fix a pair of functions in the unit spheres $f \in S_{C\left(K_{1}\right)}$ and $g \in S_{C\left(K_{2}\right)}$, we put $f_{k}^{\prime}:=$ $\left(1-|f|^{p}\right)^{1 / p} f_{k}, g_{k}^{\prime}:=\left(1-|g|^{q}\right)^{1 / q} g_{k}$ for each $1 \leq k \leq n$ and $f_{n+1}^{\prime}:=f, g_{n+1}^{\prime}:=g$.

We have $\sum_{k=1}^{n+1}\left|f_{k}^{\prime}\right|^{p} \leq 1$ and $\sum_{k=1}^{n+1}\left|g_{k}^{\prime}\right|^{q} \leq 1$. Since $\nu(|f|, 1) \geq \nu(|f|,|g|)$ and $\nu(1,|g|) \geq$ $\nu(|f|,|g|)$,

$$
0 \leq \nu\left(\left(1-|f|^{p}\right),\left(1-|g|^{q}\right)\right)=1-\left(\nu\left(|f|^{p}, 1\right)+\nu\left(1,|g|^{q}\right)-\nu\left(|f|^{p},|g|^{q}\right)\right)=1-a
$$

and so $0 \leq a \leq 1$. Thus, we obtain

$$
\begin{aligned}
\|T(f, g)\|^{r} & \leq 1-\sum_{k=1}^{n}\left\|T\left(f_{k}^{\prime}, g_{k}^{\prime}\right)\right\|^{r} \leq 1-\nu\left(\left(1-|f|^{p}\right)^{1 / p},\left(1-|g|^{q}\right)^{1 / q}\right)^{r} \\
& \leq 1-\nu\left(\left(1-|f|^{p}\right),\left(1-|g|^{q}\right)\right)^{r} \leq 1-\left|1-\nu\left(|f|^{p}, 1\right)-\nu\left(1,|g|^{q}\right)+\nu\left(|f|^{p},|g|^{q}\right)\right|^{r} \\
& \leq r\left(\nu\left(|f|^{p}, 1\right)+\nu\left(1,|g|^{q}\right)-\nu\left(|f|^{p},|g|^{q}\right)\right) \leq r\left(\nu\left(|f|^{p}, 1\right)+\nu\left(1,|g|^{q}\right)\right) .
\end{aligned}
$$

Since $f \mapsto \nu(f, 1)$ and $g \mapsto \nu(1, g)$ define positive continuous functionals on $C\left(K_{1}\right)$ and $C\left(K_{2}\right)$, respectively, we obtain the required probability Borel measures by the Riesz Theorem via adaptation of a well known approximation argument that uses weak* compactness of the closed unit ball in $C(K)^{*}$ and the measures obtained for each $n \in \mathbb{N}$ with the argument above. We leave details to the reader and refer to [10, pp. 202-203], where the linear case is considered.

We conclude our discussion with some results concerning factorization of positive bilinear operators. As we have already mentioned, if $1 \leq p<q<\infty$ and $\mu$ is a probability Borel measure on $K$, the natural canonical inclusion $i: C(K) \rightarrow L_{q, 1}(\mu)$ is $(q, p)$-summing. This allows us to give the following equivalent form of the Pisier's Factorization Theorem: an operator $T$ from a $C(K)$-space to a Banach space $Y$ is $(q, p)$-summing if and only if there is a positive operator $P: C(K) \rightarrow L$ on a Banach lattice $L$ that $i s(q, p)$-summing and an operator $S: L \rightarrow Y$ such that $T=S \circ P$. In this part we will give a complete characterization of the bilinear operators that satisfy this positive Pisier's type factorization. We have proved 
in Proposition 3.2 that this class cannot coincide with the $(r ; p, q)$-summing bilinear operators. However, the class of operators satisfying this factorization is in a sense near to the class of the $(r ; p, q)$-summing multilinear maps.

We recall that for every bilinear mapping $A: X \times Y \rightarrow Z$ there exists a unique linear mapping (called a linearization of $A) \tilde{A}: X \otimes Y \rightarrow Z$ such that $A(x, y)=\tilde{A}(x, y)$ for all $x \in x, y \in Y$. Let $K_{1}$ and $K_{2}$ be two compact Hausdorff spaces. To state the next result we need to introduce the following definition: a bilinear operator $T$ from the product $C\left(K_{1}\right) \times$ $C\left(K_{2}\right)$ to a Banach space $Y$ is norm-order dominated by a positive bilinear operator $T_{0}$ from $C\left(K_{1}\right) \times C\left(K_{2}\right)$ to a Banach lattice $E$ whenever

$$
\|\widetilde{T} z\|_{Y} \leq \sum_{k=1}^{m}\left\|T_{0}\left(\left|f_{k}\right|,\left|g_{k}\right|\right)\right\|_{E}
$$

for all $z \in C\left(K_{1}\right) \otimes C\left(K_{2}\right)$ and any choice of finite sequences $\left(f_{k}\right)_{k=1}^{m}$ in $C\left(K_{1}\right)$ and $\left(g_{k}\right)_{k=1}^{m}$ in $C\left(K_{2}\right)$ such that $|z| \leq \sum_{k=1}^{m}\left|f_{k}\right|\left|g_{k}\right|$.

Theorem 3.4. Assume that $1 \leq r, p, q<\infty$ and $1 / r \leq 1 / p+1 / q$. The following are equivalent statements about Banach space valued bilinear operator $T: C\left(K_{1}\right) \times C\left(K_{2}\right) \rightarrow Y$.

(i) $T$ is norm-order dominated by an $(r ; p, q)$-summing and positive operator.

(ii) $T$ admits a factorization:

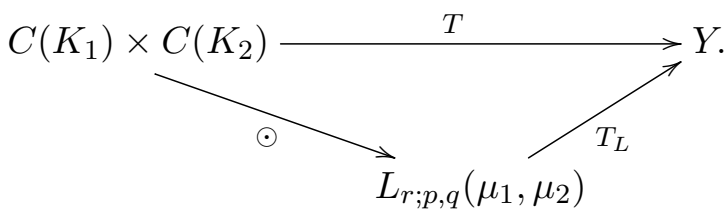

(iii) $T$ admits a factorization:

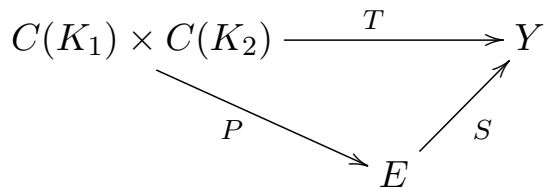

where $E$ is a Banach lattice and the factor $P$ is a positive $(r ; p, q)$-summing bilinear operator and $S$ is a bounded operator.

Proof. (i) $\Rightarrow$ (ii). Assume that the operator $T$ is norm-order dominated a positive bilinear operator $T_{0}$ from $C\left(K_{1}\right) \times C\left(K_{2}\right)$ to a Banach lattice $E$. By Theorem 3.3, it follows that there are probability Borel measures $\mu_{1}, \mu_{2}$ on $K_{1}$ and $K_{2}$, respectively, such that for every $\sum_{k=1}^{m} f_{k} \cdot g_{k} \in C\left(K_{1}\right) \otimes C\left(K_{2}\right)$, the linearization $\widetilde{T}$ of $T$ satisfies,

$$
\begin{aligned}
\left\|\widetilde{T}\left(\sum_{k=1}^{m} \odot\left(f_{k}, g_{k}\right)\right)\right\|_{Y} \leq \sum_{k=1}^{m}\left\|T_{0}\left(\left|\tilde{f}_{k}\right|,\left|\tilde{g_{k}}\right|\right)\right\|_{E} \\
\quad \leq C \sum_{k=1}^{m}\left(\left\|\tilde{f}_{k}\right\|_{L^{p}\left(K_{1}, \mu_{1}\right)}^{p / r}\left\|\tilde{f}_{k}\right\|_{C\left(K_{1}\right)}^{1-p / r}\left\|\tilde{g_{k}}\right\|_{C\left(K_{2}\right)}+\left\|\tilde{g_{k}}\right\|_{L^{q}\left(K_{2}, \mu_{2}\right)}^{q / r}\left\|\tilde{g_{k}}\right\|_{C\left(K_{2}\right)}^{1-q / r}\left\|\tilde{f}_{k}\right\|_{C\left(K_{1}\right)}\right) .
\end{aligned}
$$

This shows that $\widetilde{T}$ can be defined from $L_{r ; p, q}\left(\mu_{1}, \mu_{2}\right)$. Taking into account the continuous inclusion $C\left(K_{1}\right) \otimes_{\pi_{r ; p, q}} C\left(K_{2}\right) \hookrightarrow L_{r ; p, q}\left(\mu_{1}, \mu_{2}\right)$, we obtain the desired factorization.

(ii) $\Rightarrow$ (i). Assume that $T$ admits a factorization as in (ii). Notice that the map $\odot$ is positive and that the factorization assumed in (ii) implies the one in (iii) of Theorem 3.3. Consequently, $\odot$ is $(r ; p, q)$-summing. We need only to show that $T$ is norm-order dominated 
by $\odot$; to do this take $z \in C\left(K_{1}\right) \otimes C\left(K_{2}\right)$ and two finite sequences $\left(\tilde{f}_{j}\right)_{k=1}^{m}$ and $\left(\tilde{g}_{j}\right)_{k=1}^{m}$ in $C\left(K_{1}\right)$ and $C\left(K_{2}\right)$, respectively such that $|z| \leq \sum_{k=1}^{m}\left|\tilde{f}_{k}\right|\left|\tilde{g}_{k}\right|$. Then the lattice property of the norm of $L_{r ; p, q}\left(\mu_{1}, \mu_{2}\right)$ gives

$$
\begin{aligned}
\|\widetilde{T} z\| & \leq\|\widetilde{T}\|\|z\|_{L_{r ; p, q}\left(\mu_{1}, \mu_{2}\right)} \leq\|\widetilde{T}\|\left\|\sum _ { k = 1 } ^ { m } \left|\tilde{f}_{k}\left\|\tilde{g}_{k} \mid\right\|_{L_{r ; p, q}\left(\mu_{1}, \mu_{2}\right)}\right.\right. \\
& \leq\left\|T_{L}\right\| \sum_{k=1}^{m}\left\|\odot\left(\left|\tilde{f}_{k}\right|,\left|\tilde{g}_{k}\right|\right)\right\|_{L_{r ; p, q}\left(\mu_{1}, \mu_{2}\right)} .
\end{aligned}
$$

Thus the statement holds for $T_{0}:=\odot$.

(ii) $\Rightarrow$ (iii). The implication is an immediate consequence of the lattice structure of the space $L_{r ; p, q}\left(\mu_{1}, \mu_{2}\right)$ and the fact that $\odot$ is in this case positive and $(r ; p, q)$-summing. The converse holds directly using the characterization of positive $(r ; p, q)$-summing bilinear operators given in Theorem 3.3.

\section{REFERENCES}

[1] R. Alencar, Multilinear mappings of nuclear and integral type, Proc. Amer. Math. Soc. 94 (1985), no. 1, $33-38$.

[2] R. Alencar and M. C. Matos, Some classes of multilinear mappings between Banach spaces, Publicaciones del Departamento de Análisis Matemático 12, Universidad Complutense, Madrid, 1989.

[3] C. D. Aliprantis and O. Burkinshaw, Positive operators, Academic Press, New York and London, 1985.

[4] F. Bernicot and P. Germain, Bilinear oscillatory integrals and boundedness for new bilinear multipliers, Adv. Math. 225 (2010), no. 4, 1739-1785.

[5] R. C. Blei, Multi-linear measure theory and the Grothendieck factorizacion theorem, Proc. London Math. Soc. 56 (1988), no. 3, 529-546.

[6] G. Botelho, D. Pellegrino and P. Rueda, A unified Pietsch domination theorem, J. Math. Anal. Appl. 365 (2010), no. 1, 269-276.

[7] A. Defant and K. Floret, Tensor norms and operator ideals, North-Holland Mathematics Studies, 176. North-Holland Publishing Co., Amsterdam, 1993.

[8] A. Defant and M. Mastyło, Interpolation of Fremlin tensor products and Schur factorization of matrices, J. Funct. Anal. 262 (2012), 3981-3999.

[9] J. Diestel, J. H. Fourie and J. Swart, The metric theory of tensor products, Grothendieck's résumé revisited. American Mathematical Society, Providence, RI, 2008.

[10] J. Diestel, H. Jarchow and A. Tonge, Absolutely summing operators, Cam. Univ. Pres, Cambridge 1995.

[11] D. H. Fremlin, Tensor products of Banach lattices, Math. Ann. 211 (1974), 87-106.

[12] S. Geiss, Ideale multilinearer Abbildungen. Diplomarbeit, Jena, 1984.

[13] L. Grafakos and M. Peloso, Bilinear Fourier integral operators, J. Pseudo-Differ. Oper. Appl. 1 (2010), no. 2, 161-182.

[14] L. Grafakos and R. H. Torres, Multilinear Calderón-Zygmund theory, Adv. Math. 165 (2002), no. 1, 124-164.

[15] W. B. Johnson, H. König, B. Maurey and J. R. Retherford, Eigenvalues of p-summing and $l_{p}$-type operators in Banach spaces, J. Funct. Anal. 32 (1979), no. 3, 353-380.

[16] S. V. Kislyakov, Banach spaces and classical harmonic analysis; in: Handbook of the Geometry of Banach Spaces, Elsevier. Amsterdam. 2001.

[17] H. König, Eigenvalue Distribution of Compact Operators, Birkhäuser, Basel, 1986.

[18] J. E. Littlewood, On bounded bilinear forms in an infinite number of variables, Quart. J. Math. 2 (1930), 164-174.

[19] M. Mastyło and E. A. Sánchez Pérez, Domination and factorization of multilinear operators, J. Convex Anal. 20 (2013), no. 4, 999-1012.

[20] M. Mastyło and E. A. Sánchez Pérez, Factorization of operators through Orlicz spaces, Bull. Malays. Math. Sci. Soc. 40 (2017) 1653-1675.

[21] M. C. Matos, On multilinear mappings of nuclear type, Rev. Mat. Comput. 6 (1993), 61-81.

[22] S. Montgomery-Smith and P. Saab, p-summing operators on injective tensor products of spaces, Proc. Roy. Soc. Edinburgh Sect. A 120 (1992), no. 3-4, 283-296. 
[23] X. Mujica, $\tau(p ; q)$-summing mappings and the domination theorem, Port. Math. (N.S.) 65 (2008), no. 2, 211-226.

[24] D. Pellegrino, P. Rueda and E. A. Sánchez Pérez, Surveying the spirit of absolute summability on multilinear operators and homogeneous polynomials, RACSAM, 110 (2016), no. 1, 285-302.

[25] D. Pellegrino and J. Santos Absolutely summing multilinear operators: a panorama, Quaest. Math. 34 (2011), no. 4, 447-478.

[26] A. Pełczyński, Banach Spaces of Analytic Functions and Absolutely Summing Operators, Conf. Board of Math. Sciences, Vol. 30, Amer. Math. Soc. (1977).

[27] D. Pérez García and I. Villanueva, Multiple summing operators on $C(K)$ spaces, Ark. Mat., 42 (2004), $153-171$.

[28] A. Pietsch, Absolut p-summierende Abbildungen in normieten Räumen, Studia Math. 27 (1967), 333-353.

[29] A. Pietsch, Operator Ideals, North Holland, Amsterdam, 1980.

[30] A. Pietsch, Ideals of multilinear functionals (designs of a theory), Proceedings of the second international conference on operator algebras, ideals, and their applications in theoretical physics (Leipzig, 1983), 185-199, Teubner-Texte Math., 67, Teubner, Leipzig, 1984.

[31] G. Pisier, Factorization of operators through $L_{p \infty}$ or $L_{p 1}$ and noncommutative generalizations, Math. Ann. 276 (1986), no. 1, 105-136.

[32] G. Pisier, Grothendieck's theorem, past and present, Bull. Amer. Math. Soc. (N.S.) 49 (2012), no. 2, $237-323$.

[33] M.S. Ramanujan and E. Schock, Operator ideals and spaces of bilinear operators, Linear Multilinear Algebra 18 (1985), no. 4, 307-318.

[34] P. Rueda, E. A. Sánchez-Pérez and A. Tallab, Traced tensor norms and multiple summing multilinear operators, Linear Multilinear Algebra 65 (2017), 768-786.

[35] I. Villanueva, Integral mappings between Banach spaces, J. Math. Anal. Appl. 279 (2003), no. 1, 56-70.

M. Mastylo, Faculty of Mathematics and Computer Science, Adam Mickiewicz University in Poznań, Umultowska 87, 61-614 Poznań, Poland

E-mail address: mastylo@amu.edu.pl

E. A. Sánchez Pérez, Instituto Universitario de Matemática Pura y Aplicada, Universitat

Politècnica de València, Camino de Vera s/n, 46022 Valencia, Spain

E-mail address: easancpe@mat.upv.es 